\title{
Post-inflationary thermal histories and the refractive index of relic gravitons
}

\author{
Massimo Giovannini* \\ Department of Physics, CERN, 1211 Geneva 23, Switzerland and INFN, \\ Section of Milan-Bicocca, 20126 Milan, Italy
}

(Received 5 June 2018; revised manuscript received 9 October 2018; published 12 November 2018)

\begin{abstract}
We investigate the impact of the post-inflationary thermal histories on the cosmic graviton spectrum caused by the inflationary variation of their refractive index. Depending on the frequency band, the spectral energy distribution can be mildly red, blue, or even violet. Wide portions of the parameter space lead to potentially relevant signals both in the audio range (probed by the advanced generation of terrestrial interferometers) and in the $\mathrm{mHz}$ band (where space-borne detectors could be operational within the incoming score year). The description of the refractive index in conformally related frames is clarified.
\end{abstract}

DOI: $10.1103 /$ PhysRevD.98.103509

\section{INTRODUCTION}

Stochastic backgrounds of cosmological origin have been suggested more than forty years ago [1-3] as a genuine general relativistic effect in curved space-times. Since the evolution of the tensor modes of the geometry is not Weylinvariant [1], the corresponding classical and quantum fluctuations can be amplified not only in an anisotropic metric but also in conformally flat background geometries [2,3] (see also [4]). For this reason, backgrounds of relic gravitons are expected, with rather different properties, in a variety of cosmological scenarios and, in particular, during an isotropic phase of quasi-de Sitter expansion [5]. The backgrounds of cosmic gravitons are analyzed in terms of the spectral energy distribution in critical units, conventionally denoted by $\Omega_{g w}\left(\nu, \tau_{0}\right)$ where $\tau_{0}$ is the present value of the conformal time coordinate and $\nu$ is the comoving frequency whose numerical value coincides (at $\tau_{0}$ ) with the value of the physical frequency. ${ }^{1}$ The transition from the radiation-dominated to the matter stage of expansion leads to the infrared branch of the spectrum ranging between the $\mathrm{aHz}$ and $100 \mathrm{aHz}$ [6-8]. The standard prefixes shall be used throughout, i.e., $1 \mathrm{aHz}=10^{-18} \mathrm{~Hz}, 1 \mathrm{mHz}=10^{-3} \mathrm{~Hz}$, $1 \mathrm{MHz}=10^{6} \mathrm{~Hz}$, and so on and so forth.

Between few $\mathrm{aHz}$ and $100 \mathrm{aHz}$, the low frequency branch of the spectrum is universal and it is caused by the tensor modes of the geometry reentering after

\footnotetext{
*massimo.giovannini@cern.ch

${ }^{1}$ In this investigation the scale factor is normalized as $a\left(\tau_{0}\right)=a_{0}=1$. Natural units $\hbar=c=1$ will be used throughout.

Published by the American Physical Society under the terms of the Creative Commons Attribution 4.0 International license. Further distribution of this work must maintain attribution to the author(s) and the published article's title, journal citation, and DOI.
}

matter-radiation equality. For higher frequencies the spectral energy distribution bears the mark of the evolution of the Hubble rate prior to the radiation-dominated epoch. The simplest possibility (so far consistent with observational data) is that a quasi-de Sitter phase of expansion is followed by a radiation-dominated stage: in this case the spectral energy density is quasiflat [5,9-11] between $100 \mathrm{aHz}$ and $100 \mathrm{MHz}$. Neglecting all possible complications (damping of the tensor modes due to neutrinos [12,13], evolution of relativistic species $[14,15]$, and late-time dominance of the dark energy [15]) we can estimate ${ }^{2}$ the typical amplitude of the spectral energy distribution in critical units which is $h_{0}^{2} \Omega_{g w}\left(\nu, \tau_{0}\right) \leq \mathcal{O}\left(10^{-16.5}\right)$ for frequencies ranging between the $\mathrm{mHz}$ and $10 \mathrm{kHz}$. This minute result follows from the absolute normalization of the spectral energy distribution fixed by the upper limit on the tensor to scalar ratio $r_{T}\left(\nu_{p}\right)$ at the pivot frequency ${ }^{3} \nu_{p}=k_{p} /(2 \pi)=3.092 \mathrm{aHz}$.

In spite of the fact that the combination of different cosmic microwave background (CMB in what follows) observations imply various sets of upper bounds on $r_{T}\left(\nu_{p}\right)$, here we shall be enforcing the limit $r_{T}<0.06$ for the tensor spectral index. This limit follows from a joint analysis of Planck and BICEP2/Keck array data [16] (see also [17]). However, in a less conservative perspective we could require that $r_{T}\left(\nu_{p}\right)<0.17$, as demanded by the WMAP9 results $[18,19]$. This particular figure holds if the WMAP9 data are combined with the baryon acoustic oscillation data [20], with the South Pole Telescope data [21], and with the Atacama Cosmology Telescope data [22]. The WMAP9

\footnotetext{
${ }^{2}$ Note that $h_{0}$ is the present value of the Hubble rate $H_{0}$ in units of $100 \mathrm{~km} /(\mathrm{sec} \times \mathrm{Mpc})$.

${ }^{3}$ The scalar and tensor power spectra are customarily assigned at a pivot frequency that is largely conventional. In the present analysis we shall be dealing with a pivot wave number $k_{p}=$ $0.002 \mathrm{Mpc}^{-1}$ corresponding to a pivot frequency $\nu_{p}$.
} 
data (combined with further data sets) lead to bounds on $r_{T}\left(\nu_{p}\right)$ that are grossly similar, for the present ends, to the Planck Explorer data suggesting $r_{T}\left(\nu_{p}\right)<0.11$ [23].

One of the tacit (but key) assumptions of the concordance scenario is that radiation dominates almost suddenly after the end of inflation. This assumption is used, among other things, to assess the maximal number of inflationary $e$-folds today accessible by $\mathrm{CMB}$ observations. It is, however, not unreasonable to presume that in its early stages the Universe passed through different rates of expansion, deviating from the radiation-dominated evolution. The slowest possible rate of expansion occurs when the sound speed of the medium coincides with the speed of light [24] (see also [25]). Expansion rates even slower than the ones of the stiff phase can only be realized when the sound speed exceeds the speed of light. This possibility is, however, not compatible with the standard notion of causality. The plausible range for the existence of such a phase is between the end of inflation and the formation of the light nuclei [26-28]. If the dominance of radiation is to take place already by the time of formation of the baryon asymmetry, then the onset of radiation dominance increases from few $\mathrm{MeV}$ to the $\mathrm{TeV}$ range. In particular, if the postinflationary plasma is dominated by a stiff source (i.e., characterized by a barotropic index $w=p / \rho$ larger than $1 / 3$ ) the corresponding spectral energy density inherits a blue (or even violet) slope for typical frequencies larger than the $\mathrm{mHz}$ and anyway smaller than $100 \mathrm{GHz}$. In this case, depending on the parameters characterizing the stiff evolution, the spectral energy distribution can be of the order of $10^{-10}$ in the audio band while in the $\mathrm{mHz}$ band is at most $10^{-15}$.

In quintessence scenarios the present dominance of a cosmological term is translated into the late-time dominance of the potential of a scalar degree of freedom (d.o.f.) that is called quintessence (see, e.g., [29]). If we also demand the existence of an early inflationary phase accounting for the existence of large-scale inhomogeneities, the inflaton potential must dominate at early times while the quintessence potential should be relevant much later (see the second and third papers in Refs. [26,27]). In between, the scalar kinetic term of the inflaton/quintessence field dominates the background. When the inflaton and the quintessence field are identified, the existence of this phase is explicitly realized [27] even if a similar phenomenon may take place also in slightly different scenarios.

Gravitational waves might acquire an effective index of refraction when they travel in curved space-times [30,31] and this possibility has been recently revisited by studying the parametric amplification of the tensor modes of the geometry during a quasi-de Sitter stage of expansion [32]: when the refractive index mildly increases during inflation, the corresponding speed of propagation of the waves diminishes and the power spectra of the relic gravitons are then blue, i.e., tilted towards high frequencies.
The purpose of this paper is to compute the spectral energy distribution of the relic gravitons produced by a dynamical refractive index without assuming a standard post-inflationary thermal history.

Even though the current upper limits on stochastic backgrounds of relic gravitons are still far from their final targets [33,34], the advanced Ligo/Virgo projects are described in $[35,36]$. We shall then suppose, according to Refs. $[35,36]$, that the terrestrial interferometers (in their advanced version) will be one day able to probe chirp amplitudes $\mathcal{O}\left(10^{-25}\right)$ corresponding to spectral amplitudes $h_{0}^{2} \Omega_{g w}=\mathcal{O}\left(10^{-11}\right)$. In the foreseeable future there should be at least one supplementary interferometer operational in the audio band, namely, the Japanese Kamioka Gravitational Wave Detector (for short Kagra) $[37,38]$ which is, in some sense, the prosecution and the completion of the Tama-300 experiment [39]. In the class of wide-band detectors we should also mention the GEO-600 experiment [40] (which is now included in the Ligo/Virgo consortium [41]) and the Einstein telescope [42] whose sensitivities should definitively improve on the advanced Ligo/Virgo targets.

The target sensitivity to detect the stochastic background of inflationary origin should correspond to a chirp amplitude $h_{c}=\mathcal{O}\left(10^{-29}\right)$ (or smaller) and to a spectral energy distribution in critical units $h_{0}^{2} \Omega_{g w}=\mathcal{O}\left(10^{-16}\right)$ (or smaller). These orders of magnitude estimates directly come from the amplitude of the quasiflat plateau produced in the context of single-field inflationary scenarios; in this case the plateau encompasses the $\mathrm{mHz}$ and the audio bands with basically the same amplitude. Even though these sensitivities are beyond reach for the current interferometers, a number of ambitious projects will be supposedly operational in the future. The space-borne interferometers, such as (e)Lisa (Laser Interferometer Space Antenna) [43], Bbo (Big Bang Observer) [44], and Decigo (Deci-hertz Interferometer Gravitational Wave Observatory) [45,46], might operate between a few $\mathrm{mHz}$ and the $\mathrm{Hz}$ hopefully within the following score year. While the sensitivities of these instruments are still very hypothetical, we can suppose (with a certain dose of optimism) that they could even range between $h_{0}^{2} \Omega_{g w}=\mathcal{O}\left(10^{-12}\right)$ and $h_{0}^{2} \Omega_{g w}=\mathcal{O}\left(10^{-15}\right)$.

The layout of the paper is the following. In Sec. II we shall introduce the main technical aspects of the problem and we shall also analyze the evolution of the effective horizon and the amplification of the relic gravitons. Section III will be focused on the analytic (though approximate) estimates of the graviton spectra while Sec. IV contains the discussion of the detectability prospects. Some concluding remarks are collected in Sec. V. Since the action of the gravitons depends on the specific frame, different (conformally related) frames lead to actions that are superficially different but ultimately equivalent. This discussion (as well as other aspects pertaining to the same theme) will be relegated to the Appendices. 


\section{REFRACTIVE INDEX AND EFFECTIVE HORIZON}

\section{A. Canonical action}

The evolution of the tensor modes of the geometry in the presence of a dynamical refractive index $n(\tau)$ can be parametrized in the following manner:

$S=\frac{1}{8 \ell_{P}^{2}} \int d^{3} x \int d \tau a^{2}\left[\partial_{\tau} h_{i j} \partial_{\tau} h_{i j}-\frac{1}{n^{2}} \partial_{k} h_{i j} \partial_{k} h_{i j}\right]$.

In the case $n \rightarrow 1$ the action of Eq. (2.1) reproduces the original Ford and Paker result [3]. Equation (2.1) will be referred to as the canonical action for the relic gravitons in the presence of a refractive index. In this section we shall show that this action can be quantized even though the presence of the refractive index imposes some technical differences. From the physical viewpoint the most notable aspect is that the modes of the gravitational field are amplified because of the presence of an effective horizon whose specific form is affected by the evolution of the refractive index, as we shall show hereunder.

It is convenient to simplify the action Eq. (2.1) by introducing a generalized time coordinate, conventionally denoted by $\eta$ :

$$
n(\eta) d \eta=d \tau, \quad b(\eta)=\frac{a}{\sqrt{n}} .
$$

With the redefinition (2.2) of the time coordinate, Eq. (2.1) can be rewritten as

$S=\frac{1}{8 \ell_{P}^{2}} \int d^{3} x \int d \eta b^{2}(\eta)\left[\partial_{\eta} h_{i j} \partial_{\eta} h_{i j}-\partial_{k} h_{i j} \partial_{k} h_{i j}\right]$.

The function $b(\eta)$ plays the role of an effective scale factor: note, in fact, that in the limit $n \rightarrow 1$ we have that $\eta$ coincides with $\tau$ and that, consequently, $b(\eta) \rightarrow a(\tau)$. When $n \neq 1$ the evolution of $b(\eta)$ defines an effective horizon, namely,

$$
\mathcal{F}=\frac{\dot{b}}{b}, \quad \dot{b}=\frac{\partial b}{\partial \eta}=\frac{1}{n} \frac{\partial b}{\partial \tau} \equiv \frac{b^{\prime}}{n},
$$

where the prime denotes a derivation with respect to the conformal time coordinate $\tau$ while the overdot denotes a derivation with respect to the $\eta$ time (and not a derivation with respect to the cosmic time coordinate, as in the conventional notations). To clarify this point and to avoid potential confusions the following relations are explicitly given:

$$
\begin{array}{rlrl}
\mathcal{F} & =\frac{\dot{b}}{b}=\frac{\partial \ln b}{\partial \eta} \equiv n a F, & F=\frac{\partial \ln b}{\partial t}, \\
\mathcal{H}=\frac{a^{\prime}}{a}=\frac{\partial \ln a}{\partial \tau} \equiv a H, & H=\frac{\partial \ln a}{\partial t},
\end{array}
$$

which can be verified by using Eq. (2.2) and the relation of $\tau$ the cosmic time coordinate $t$, i.e.,

$$
n(\eta) d \eta=d \tau=d t / a .
$$

The canonical action of Eq. (2.1) can also be expressed in a more covariant language or even in a different conformally related frame. In the current literature some authors expressed the canonical action of Eq. (2.1) in a conformally related frame and claimed to have a different action for the refractive index. Since this discussion is not central to the theme of this paper we relegated the relevant analysis to Appendices A and B. The interested reader can find there how the action (2.1) is modified in a different conformally related frame.

\section{B. The canonical Hamiltonian and the mode functions}

From the canonical action of Eq. (2.3) we can deduce the canonical Hamiltonian. In terms of the canonical normal modes $\mu_{i j}(\vec{x}, \eta)=b(\eta) h_{i j}(\vec{x}, \eta)$, Eq. (2.3) becomes

$$
\begin{aligned}
S= & \frac{1}{8 \ell_{P}^{2}} \int d^{3} x \int d \eta\left[\left(\partial_{\eta} \mu_{i j}\right)\left(\partial_{\eta} \mu_{i j}\right)-2 \mathcal{F}\left(\partial_{\eta} \mu_{i j}\right) \mu_{i j}\right. \\
& \left.-\left(\partial_{k} \mu_{i j}\right)\left(\partial_{k} \mu_{i j}\right)\right] .
\end{aligned}
$$

Up to a total time derivative, Eq. (2.8) can also be written as

$$
\begin{aligned}
S= & \frac{1}{8 \ell_{P}^{2}} \int d^{3} x \int d \eta\left[\left(\partial_{\eta} \mu_{i j}\right)\left(\partial_{\eta} \mu_{i j}\right)-\left(\dot{\mathcal{F}}+\mathcal{F}^{2}\right) \mu_{i j} \mu_{i j}\right. \\
& \left.-\left(\partial_{k} \mu_{i j}\right)\left(\partial_{k} \mu_{i j}\right)\right] .
\end{aligned}
$$

Since $\mu_{i j}(\vec{x}, \eta)$ is given as the sum over the two polarizations $\oplus$ and $\otimes$

$$
\mu_{i j}=\sqrt{2} e_{P} \sum_{\lambda=\oplus, \otimes} e_{i j}^{(\lambda)} \mu_{\lambda}, \quad e_{i j}^{(\lambda)} e_{i j}^{\left(\lambda^{\prime}\right)}=2 \delta^{\left(\lambda \lambda^{\prime}\right)},
$$

the action (2.9) becomes immediately

$$
\begin{gathered}
S=\int d \eta L(\eta), \quad L(\eta)=\sum_{\lambda=\oplus, \otimes} \int d^{3} x \mathcal{L}_{\lambda}(\vec{x}, \eta), \\
\mathcal{L}_{\lambda}=\frac{1}{2}\left[\dot{\mu}_{\lambda}^{2}-\left(\dot{\mathcal{F}}+\mathcal{F}^{2}\right) \mu_{\lambda}^{2}-\left(\partial_{k} \mu_{\lambda}\right)^{2}\right] .
\end{gathered}
$$

From Eqs. (2.11) and (2.12) the canonical momenta are $\pi_{\lambda}=\dot{\mu}_{\lambda}$; consequently the canonical Hamiltonian associated with Eqs. (2.11) and (2.12) is given by

$$
\begin{aligned}
H(\eta) & =\sum_{\lambda=\oplus, \otimes} H_{\lambda}(\eta) \\
H_{\lambda} & =\frac{1}{2} \int d^{3} x\left[\pi_{\lambda}^{2}+\left(\dot{\mathcal{F}}+\mathcal{F}^{2}\right) \mu_{\lambda}^{2}+\left(\partial_{k} \mu_{\lambda}\right)^{2}\right] .
\end{aligned}
$$


The commutation relations at equal $\eta$ times

$$
\left[\hat{\mu}_{\lambda}(\vec{x}, \eta), \hat{\pi}_{\lambda^{\prime}}(\vec{y}, \eta)\right]=i \delta^{(3)}(\vec{x}-\vec{y}) \delta_{\lambda \lambda^{\prime}}
$$

together with the explicit form of the Hamiltonian (2.13), lead directly to the evolution equations of the operators $\hat{\mu}_{\lambda}$ and $\hat{\pi}_{\lambda}$ :

$$
\partial_{\eta} \hat{\mu}_{\lambda}=\hat{\pi}_{\lambda}, \quad \partial_{\eta} \hat{\pi}_{\lambda}=\left(\mathcal{F}^{2}+\dot{\mathcal{F}}\right) \hat{\mu}_{\lambda}+\nabla^{2} \hat{\mu}_{\lambda} .
$$

The Fourier representations of $\hat{\mu}_{\lambda}$ and $\hat{\pi}_{\lambda}$ are

$$
\begin{aligned}
& \hat{\mu}_{\lambda}(\vec{x}, \eta)=\frac{1}{(2 \pi)^{3 / 2}} \int d^{3} k\left[\hat{a}_{\vec{k}, \lambda} f_{k, \lambda} e^{-i \vec{k} \cdot \vec{x}}+\hat{a}_{\vec{k}, \lambda}^{\dagger} f_{k, \lambda}^{*} e^{i \vec{k} \cdot \vec{x}}\right], \\
& \hat{\pi}_{\lambda}(\vec{x}, \eta)=\frac{1}{(2 \pi)^{3 / 2}} \int d^{3} k\left[\hat{a}_{\vec{k}, \lambda} g_{k, \lambda} e^{-i \vec{k} \cdot \vec{x}}+\hat{a}_{\vec{k}, \lambda}^{\dagger} g_{k, \lambda}^{*} e^{i \vec{k} \cdot \vec{x}}\right],
\end{aligned}
$$

where $\left[a_{\vec{k}, \lambda}, \hat{a}_{\vec{p}, \lambda}^{\dagger}\right]=\delta^{(3)}(\vec{k}-\vec{p}) \delta_{\lambda, \lambda^{\prime}}$. The evolution of the mode functions $f_{k, \lambda}$ and $g_{k, \lambda}$ follows from Eq. (2.15) while the normalization of their Wronskian is a consequence of the commutation relations of Eq. (2.14):

$$
\begin{gathered}
\dot{f}_{k, \lambda}=g_{k, \lambda}, \quad \dot{g}_{k, \lambda}=-k^{2} f_{k, \lambda}+\left(\dot{\mathcal{F}}+\mathcal{F}^{2}\right) f_{k, \lambda}, \\
f_{k, \lambda}(\eta) f_{k, \lambda}^{*}(\eta)-f_{k, \lambda}^{*}(\eta) g_{k, \lambda}(\eta)=i .
\end{gathered}
$$

The equations for the mode functions reported in Eq. (2.18) can be decoupled as

$$
\ddot{f}_{k}+\left[k^{2}-\frac{\ddot{b}}{b}\right] f_{k}=0, \quad g_{k}=\dot{f}_{k},
$$

where the polarization index has been omitted since the result of Eq. (2.20) holds both for $\oplus$ and for $\otimes$. By recalling that $\hat{h}_{i j} b=\hat{\mu}_{i j}$, the mode expansion of the tensor amplitude $\hat{h}_{i j}(\vec{x}, \eta)$ in the $\eta$ time is given by

$$
\hat{h}_{i j}(\vec{x}, \eta)=\frac{\sqrt{2} \ell_{P}}{(2 \pi)^{3 / 2} b(\eta)} \sum_{\lambda} \int d^{3} k e_{i j}^{(\lambda)}(\vec{k})\left[f_{k, \lambda}(\eta) \hat{a}_{\vec{k} \lambda} e^{-i \vec{k} \cdot \vec{x}}+f_{k, \lambda}^{*}(\eta) \hat{a}_{\vec{k} \lambda}^{\dagger} e^{i \vec{k} \cdot \vec{x}}\right],
$$

where the explicit form of the two polarizations can be written as

$$
e_{i j}^{(\oplus)}(\hat{k})=\left(\hat{m}_{i} \hat{m}_{j}-\hat{q}_{i} \hat{q}_{j}\right), \quad e_{i j}^{(\otimes)}(\hat{k})=\left(\hat{m}_{i} \hat{q}_{j}+\hat{q}_{i} \hat{m}_{j}\right),
$$

and $\hat{k}_{i}=k_{i} /|\vec{k}|, \hat{m}_{i}=m_{i} /|\vec{m}|$ and $\hat{q}=q_{i} /|\vec{q}|$ are three mutually orthogonal directions and $\hat{k}$. If we now represent the field operator $\hat{h}_{i j}(\vec{x}, \eta)$ in Fourier space:

$$
\hat{h}_{i j}(\vec{p}, \eta)=\frac{1}{(2 \pi)^{3 / 2}} \int d^{3} x \hat{h}_{i j}(\vec{x}, \eta) e^{i \vec{p} \cdot \vec{x}}
$$

we also have from Eqs. (2.21) and (2.23):

$\hat{h}_{i j}(\vec{p}, \eta)=\frac{1}{b} \sum_{\lambda}\left[e_{i j}^{(\lambda)}(\hat{p}) f_{k, \lambda}(\eta) \hat{a}_{\vec{p} \lambda}+e_{i j}^{(\lambda)}(-\hat{p}) f_{k, \lambda}^{*}(\eta) \hat{a}_{-\vec{p} \lambda}^{\dagger}\right]$.

The two-point functions computed from Eq. (2.24) are simply ${ }^{4}$

\footnotetext{
${ }^{4}$ For the sake of notational accuracy, we remind that, throughout this analysis, natural logarithms will be denoted by $\ln$ while the common logarithms will be denoted by log.
}

$$
\begin{aligned}
& \left\langle\hat{h}_{i j}(\vec{x}, \eta) \hat{h}_{i j}(\vec{x}+\vec{r}, \eta)\right\rangle=\int d \ln k \mathcal{P}_{T}(k, \eta) j_{0}(k r), \\
& \left\langle\hat{h}_{i j}(\vec{k}, \eta) \hat{h}_{m n}(\vec{p}, \eta)\right\rangle=\frac{2 \pi^{2}}{k^{3}} \mathcal{P}_{T}(k, \eta) \mathcal{S}_{i j m n}(\hat{k}) \delta^{(3)}(\vec{k}+\vec{p}),
\end{aligned}
$$

where $j_{0}(\mathrm{kr})$ is the spherical Bessel function of zeroth order $[47,48]$. The tensor power spectrum of Eqs. (2.25) and (2.26) is then given by

$$
\begin{gathered}
\mathcal{P}_{T}(k, \eta)=\frac{4 \ell_{P}^{2}}{\pi^{2} b^{2}(\eta)} k^{3}\left|f_{k}(\eta)\right|^{2}, \\
\mathcal{S}_{i j m n}(\hat{k})=\frac{1}{4}\left[p_{m i}(\hat{k}) p_{n j}(\hat{k})+p_{m j}(\hat{k}) p_{n i}(\hat{k})-p_{i j}(\hat{k}) p_{m n}(\hat{k})\right] \\
\equiv \sum_{\lambda} e_{i j}^{(\lambda)}(\hat{k}) e_{m n}^{(\lambda)}(\hat{k}) / 4, \quad p_{i j}(\hat{k})=\left(\delta_{i j}-\hat{k}_{i} \hat{k}_{j}\right) .
\end{gathered}
$$

\section{Evolution of the effective horizon}

The variation of the refractive index can be measured in units of the Hubble rate in full analogy with what it is customarily done in the case of the slow-roll parameter, namely, 


$$
\alpha=\frac{1}{H} \frac{\partial \ln n}{\partial t}=\frac{\partial \ln n}{\partial \ln a}, \quad \epsilon=-\frac{1}{H^{2}} \frac{\partial H}{\partial t} .
$$

Equation (2.29) also implies that the evolution of $n(a)$ could be considered as piecewise continuous across a certain critical value of the scale factor $a_{*}$; more specifically the situation we are interested in is the one where

$$
n(a)=n_{i}\left(\frac{a}{a_{i}}\right)^{\alpha}, \quad a<a_{*},
$$

while $n(a)=1$ for $a>a_{*}$. It is relatively simple to imagine a number of continuous interpolation between the two regimes but what matters for the present considerations is overall the continuity of $n(a)$, not the specific form of the profile across the normalcy transition. One of the simplest possibilities is given by ${ }^{5} n(a, \xi)=n_{i}\left(a / a_{i}\right)^{\alpha} e^{-\xi a / a_{*}}+1$, going as $a^{\alpha}$ for $a<a_{*}$ and approaching 1 quite rapidly when $a>a_{*}$ and $\xi>1$. The typical scale $a_{*}$ [roughly corresponding to the maximum of $n(a)$ ] may coincide with the end of the inflationary phase but this possibility is neither generic nor compulsory. The value of $a_{*}$ corresponds to a critical number of $e$-folds $N_{*}$ which is of the order of $N_{t}$ (i.e., the total number of $e$-folds) if $a_{*}$ marks the end of the inflationary phase. This identification is, however, not mandatory and it will also be relevant, from the physical viewpoint, to consider the case $N_{*}<N_{t}$ or even $N_{*} \ll N_{t}$.

In view of the analysis of Sec. IV, it is relevant to mention that $\dot{b} \geq 0$; this means that $b(\eta)$ is always an increasing function of the $\eta$ coordinate defined in Eq. (2.2). More specifically we can express $b$ as a function of $a$ and verify that $b(a)$ always increases:

$$
b(x)=\frac{a}{\sqrt{n}} \propto \frac{x}{\sqrt{n_{*} x^{\alpha} e^{-\xi x}+1}},
$$

where $n_{*}=n_{i}\left(a_{*} / a_{i}\right)^{\alpha}$. Equation (2.31) is always increasing $^{6}$ as a function of $x=a / a_{*}$. Since we shall bound the attention to the case of expanding scale factors, Eq. (2.31) implies that the explicit evolution of $b$ (either in $\tau$ or in $\eta$ ) is always monotonically increasing.

The relations between $\eta, \tau$ and the Hubble radius during the refractive phase are affected by the value of the slowroll parameter. This is a generic consequence of Eq. (2.7)

\footnotetext{
${ }^{5}$ Note that $n_{i} \geq 1$ but we shall always consider the case $n_{i}=1$ as representative of the general situation.

${ }^{6}$ We can take, for instance, $N_{t}=\mathcal{O}(60)$ and different values of $N_{*}<N_{t}$. Since $0<\alpha<1, b(x)$ increases for $0<x<1$. Moreover $b(x)$ is also increasing for $x>1$. There can be situations where, depending on the values of the parameters, the derivative of $b$ with respect to $x$ is always positive except for a small region $x=\mathcal{O}(1)$ (i.e., $a \simeq a_{*}$ ): in these cases the derivative changes sign twice so that $b(x)$ has a local maximum and a local minimum both occurring for $x=\mathcal{O}(1)$. In spite of that, $b(x)$ always increases $x<1$ and for $x>1$.
}

that fixes the relation between $\eta$ and the conformal time coordinate:

$$
\eta=\int \frac{d \tau}{n}=\int \frac{d a}{a^{2} H n} .
$$

If we now integrate Eq. (2.32) by parts we will have

$$
\int \frac{d a}{a^{2} H n}=-\frac{1}{a H n}+\int \frac{d a}{a^{2} H n}(\epsilon-\alpha),
$$

implying, together with Eq. (2.32), that

$$
a H n=-\frac{1}{(1-\epsilon+\alpha) \eta} .
$$

The pump field $\ddot{b} / b$ of Eq. (2.20) during the refractive phase can be written as

$$
\frac{\ddot{b}}{b}=\dot{\mathcal{F}}+\mathcal{F}^{2}=n^{2} H^{2} a^{2}\left[\delta^{2}+\delta(1+\alpha-\epsilon)\right], \quad \delta=1-\frac{\alpha}{2} .
$$

Inserting Eq. (2.34) into Eq. (2.35) we finally obtain

$$
\frac{\ddot{b}}{b}=\frac{\delta^{2}+\delta(1-\epsilon+\alpha)}{(1-\epsilon+\alpha)^{2} \eta^{2}},
$$

which can also be written as

$$
\frac{\ddot{b}}{b}=\frac{\mu^{2}-1 / 4}{\eta^{2}}, \quad \mu=\frac{1}{2}+\frac{\delta}{1-\epsilon+\alpha} .
$$

The same result can be obtained by assuming a slow-roll phase

$$
b=\frac{a}{\sqrt{n}}=b_{*}\left(\frac{a}{a_{*}}\right)^{\delta}, \quad b_{*}=\frac{a_{*}}{\sqrt{n_{*}}},
$$

where $a(\tau)=\left(-\tau / \tau_{*}\right)^{-\beta}$ and $\beta=1 /(1-\epsilon)$. Thus, thanks to Eq. (2.7) we have

$$
\left(-\frac{\eta}{\eta_{*}}\right)=\left(-\frac{\tau}{\tau_{*}}\right)^{1+\alpha \beta}, \quad \eta_{*}=\frac{\tau_{*}}{n_{*}(1+\alpha \beta)} .
$$

The result of Eq. (2.39) implies

$$
b(\eta)=b_{*}\left(-\frac{\eta}{\eta_{*}}\right)^{-\nu}, \quad \nu=\frac{\delta \beta}{1+\alpha \beta} .
$$

If we now compute $\ddot{b} / b$ from Eq. (2.40) we obtain exactly the result of Eq. (2.37) where $\mu=(\nu+1 / 2)$. Recalling that $\beta=1 /(1-\epsilon)$ we have that $\nu=\delta /(1-\epsilon+\alpha)$ so that the results of Eqs. (2.37) and (2.40) coincide and are both consistent with Eq. (2.38). 


\section{COSMIC GRAVITON SPECTRA AND THERMAL HISTORIES}

The cosmic graviton spectra can be estimated analytically $[11,14,26,49,50]$ by adapting some of the standard methods customarily employed in the conventional case when the spectral index is not varying. These methods must be revisited, however, in a slightly different perspective since the evolution of $\eta$ and of the conformal time coordinate only coincide, in the present framework, after the end of inflation. In the limit $n \rightarrow 1$ we have that $\eta \rightarrow \tau$ and the solutions of the mode functions coincide with the standard ones. In this section we are going to compute the power spectra of the relic gravitons when the corresponding wavelengths are, respectively, larger and smaller than the effective horizon. In the last part of the section we shall compute the spectral energy density within different thermal histories.

We therefore start by noting that Eq. (2.20) is equivalent to an integral equation whose initial conditions are assigned at the reference time $\eta_{\mathrm{ex}}$ :

$$
\begin{aligned}
f_{k}(\eta)= & \frac{b}{b_{\mathrm{ex}}}\left\{f_{k}\left(\eta_{\mathrm{ex}}\right)+\left[\dot{f}_{k}\left(\eta_{\mathrm{ex}}\right)-\mathcal{F}_{\mathrm{ex}} f_{k}\left(\eta_{\mathrm{ex}}\right)\right] \int_{\eta_{\mathrm{ex}}}^{\eta} \frac{b_{\mathrm{ex}}^{2}}{b^{2}\left(\eta_{1}\right)} d \eta_{1}\right. \\
& \left.-k^{2} \int_{\eta_{\mathrm{ex}}}^{\eta} \frac{d \eta_{1}}{b^{2}\left(\eta_{1}\right)} \int_{\eta_{\mathrm{ex}}}^{\eta_{1}} b_{\mathrm{ex}} b\left(\eta_{2}\right) f_{k}\left(\eta_{2}\right) d \eta_{2}\right\},
\end{aligned}
$$

where $\eta_{\mathrm{ex}}$ is defined as the turning point at which the solution to Eq. (2.20) changes its analytic form:

$$
k^{2}=\frac{\ddot{b}_{\mathrm{ex}}}{b_{\mathrm{ex}}}, \quad \ddot{b}_{\mathrm{ex}} \neq 0 .
$$

Equation (3.2) can be dubbed by saying that at $\eta_{\mathrm{ex}}$ the given mode $k$ exits the effective horizon defined by the evolution of $b$; the second requirement of Eq. (3.2) is for the moment pleonastic since the exit always occurs in a regime where $\ddot{b}_{\text {ex }} \neq 0$. Even though $b(\eta)$ never evolves linearly in the vicinity of the exit, this occurrence may arise close to the reentry that defines the second relevant turning point of the problem.

\section{A. The large-scale power spectra}

Neglecting the terms $\mathcal{O}\left(k^{2} \eta^{2}\right)$, the lowest order solution of Eq. (3.1) is

$$
\begin{aligned}
f_{k}(\eta)= & \frac{b(\eta)}{b_{\mathrm{ex}}}\left\{f_{k}\left(\eta_{\mathrm{ex}}\right)+\left[\dot{f}_{k}\left(\eta_{\mathrm{ex}}\right)-\mathcal{F}_{\mathrm{ex}} f_{k}\left(\eta_{\mathrm{ex}}\right)\right]\right. \\
& \left.\times \int_{\eta_{\mathrm{ex}}}^{\eta} \frac{b_{\mathrm{ex}}^{2}}{b^{2}\left(\eta_{1}\right)} d \eta_{1}\right\}, \\
g_{k}(\eta)= & \frac{b_{\mathrm{ex}}}{b(\eta)}\left\{g_{k}\left(\eta_{\mathrm{ex}}\right)+\left[\dot{g}_{k}\left(\eta_{\mathrm{ex}}\right)+\mathcal{F}_{\mathrm{ex}} g_{k}\left(\eta_{\mathrm{ex}}\right)\right]\right. \\
& \left.\times \int_{\eta_{\mathrm{ex}}}^{\eta} \frac{b^{2}\left(\eta_{1}\right)}{b_{\mathrm{ex}}^{2}} d \eta_{1}\right\},
\end{aligned}
$$

where, according to Eq. (2.20), $\dot{f}_{k}\left(\eta_{\mathrm{ex}}\right)=g_{k}\left(\eta_{\mathrm{ex}}\right)$ and $\dot{f}_{k}(\eta)=g_{k}(\eta)$. Equations (3.3) and (3.4) determine the approximate form of the power spectrum for wavelengths larger than the Hubble radius. Since the second term appearing inside the squared bracket at the right-hand side of Eq. (3.3) is subleading for typical wavelengths larger than the effective horizon, after inserting Eq. (3.3) into Eq. (2.27) the tensor power spectrum becomes

$$
\begin{aligned}
\mathcal{P}_{T}(k, \eta) & =\frac{2 \ell_{P}^{2}}{\pi^{2} b_{*}^{2} \eta_{*}^{2}}|A|^{2}\left(-k \eta_{*}\right)^{2(1-\nu),} \\
|A| & =\sqrt{2 k}\left|f_{k}\left(\eta_{\mathrm{ex}}\right)\right|,
\end{aligned}
$$

where Eq. (2.40) has been used to get an explicit expression of $b(\eta)$ in the regime $\eta<-\eta_{*}$. The amplitude $|A|$ appearing in Eq. (3.5) parametrizes, up to an irrelevant phase, the mismatch between the exact and the approximate solutions at $\eta_{\text {ex }}$ : for $k^{2} \ll|\ddot{b} / b|$ the correctly normalized solutions of Eq. (2.20) are $f_{k}(\eta)=e^{ \pm i k \eta} / \sqrt{2 k}$. However, as soon as $\eta_{\mathrm{ex}}$ is approached the amplitude gets slightly modified and by recalling Eq. (2.37) the exact solution of Eq. (2.20) can be written in terms of Hankel functions [47,48]

$$
\begin{aligned}
f_{k}(\eta) & =\frac{\mathcal{N}}{\sqrt{2 k}} \sqrt{-k \eta} H_{\mu}^{(1)}(-k \eta), \quad \mathcal{N}=\sqrt{\frac{\pi}{2}} e^{i \pi(\mu+1 / 2) / 2}, \\
\mu & =\nu+\frac{1}{2}=\frac{3-\epsilon}{2(1+\alpha-\epsilon)},
\end{aligned}
$$

where $H_{\mu}^{(1)}(k \eta)$ is the Hankel function of the first kind. ${ }^{7}$ For wavelengths larger than the Hubble radius, the Hankel function of Eq. (3.6) can be expanded in the limit $|k \eta| \ll 1$ so that thanks to Eq. (2.27) the tensor power spectrum becomes

$$
\mathcal{P}_{T}(k, \eta)=\frac{\ell_{P}^{2} 2^{2 \mu}}{\pi^{3} b_{*}^{2} \eta_{*}^{2}} \Gamma^{2}(\mu)\left(-k \eta_{*}\right)^{3-2 \mu} .
$$

Since $3-2 \mu=2(1-\nu)$ [as implied by Eq. (3.6)], the ratio between Eqs. (3.5) and (3.7) implies that

$n_{T}=2(1-\nu), \quad \mu=\nu+\frac{1}{2}, \quad|A(\mu)|=\frac{\Gamma(\mu)}{\sqrt{\pi}} 2^{\mu-1 / 2}$,

where $\nu$ has been defined in Eq. (2.40). The value of $|A(\mu)|$ estimates the theoretical error of the treatment based on Eq. (3.5) and on the approximate form of the mode functions. While it is often plausible to neglect the complication $^{8}$ of $A(\mu)$ and simply set $A(\mu) \rightarrow 1$, at low frequencies the absolute normalization of the cosmic graviton spectrum

\footnotetext{
${ }^{7}$ Unlike the standard case the argument of the Hankel function in Eq. (3.6) is not $k \tau$ but rather $k \eta$. Recalling Eq. (2.39) the solution (3.6) is then simple in terms of $\eta$ but not in terms of $\tau$.

${ }^{8}$ This choice is practical for a swift derivation of the slopes characterizing the spectral energy distribution inside the Hubble radius.
} 
is, however, very sensitive to the value of the mode functions for $\eta=\mathcal{O}\left(\eta_{\mathrm{ex}}\right)$. It is then mandatory to use Eq. (3.7) which can also be expressed as

$$
\begin{aligned}
\mathcal{P}_{T}\left(k, \eta_{*}\right)= & \left(\frac{H_{*}}{M_{P}}\right)^{2} \frac{2^{6-n_{T}}}{\pi^{2}} \Gamma^{2}\left(\frac{3-n_{T}}{2}\right) n_{*}^{3-n_{T}} \\
& \times\left|1+\frac{\alpha}{1-\epsilon}\right|^{2-n_{T}}\left(\frac{k}{a_{*} H_{*}}\right)^{n_{T}},
\end{aligned}
$$

where $H_{*}$ denotes the Hubble rate at $\eta_{*}$. Equation (3.9) is the large-scale power spectrum valid for $k<a_{*} H_{*}$. The scales that exited the Hubble radius for $\eta>-\eta_{*}$ have a different spectral slope and, in this respect, we have a twofold possibility. If $\eta_{*}$ coincides with the end of inflation, then the power spectrum will still be given by Eq. (3.12) where, however, $N_{t}=N_{*}$. Conversely if the refractive phase terminates before the end of inflation the power spectrum will have a further branch for $a_{*} H_{*}<k \leq a_{1} H_{1}$ :

$\mathcal{P}_{T}\left(k, \eta_{*}\right)=\left(\frac{H_{1}}{M_{P}}\right)^{2} \frac{2^{6-\bar{n}_{T}}}{\pi^{2}} \Gamma^{2}\left(\frac{3-\bar{n}_{T}}{2}\right)\left(\frac{k}{a_{1} H_{1}}\right)^{\bar{n}_{T}}$,

where $\bar{n}_{T}=-2 \epsilon /(1-\epsilon)$. It is relevant to remark that in the limit $\alpha \rightarrow 0$ we have $\mu \rightarrow(3-\epsilon) /[2(1-\epsilon)]$ where $\mu$ is the Bessel index appearing in Eq. (3.6). Equation (3.10) can be further modified by appreciating that since between $-\eta_{*}$ and $-\tau_{1}$ the background inflates and we have

$H_{*} a_{*}=\left(H_{1} a_{1}\right) e^{N_{*}-N_{t}}, \quad n_{*}=n_{i}\left(a_{*} / a_{i}\right)^{\alpha} \equiv n_{i} e^{\alpha N_{*}}$.

Taking into account Eqs. (3.10) and (3.11) the power spectrum (3.9) finally becomes

$$
\begin{gathered}
\mathcal{P}_{T}\left(k, \tau_{1}\right)=\left(\frac{H_{1}}{M_{P}}\right)^{2} q_{T}\left(n_{i}, N_{t}, N_{*}, n_{T}\right) \\
\times\left|1+\frac{\alpha}{1-\epsilon}\right|^{2-n_{T}}\left(\frac{k}{a_{1} H_{1}}\right)^{n_{T}}, \\
q_{T}\left(n_{i}, N_{t}, N_{*}, n_{T}\right) \\
=\frac{2^{6-n_{T}}}{\pi^{2}} \Gamma^{2}\left(\frac{3-n_{T}}{2}\right) n_{i}^{3-n_{T}} e^{\alpha N_{*}\left(3-n_{T}\right)-n_{T}\left(N_{*}-N_{t}\right)},
\end{gathered}
$$

where $M_{P}=\sqrt{8 \pi} \bar{M}_{P}$ [see also the definitions after Eq. (A1)]. Equation (3.12) determines the tensor to scalar ratio whose explicit form is

$r_{T}(k)=\frac{\epsilon}{\pi} q_{T}\left(n_{i}, N_{t}, N_{*}, n_{T}\right)\left|1+\frac{\alpha}{1-\epsilon}\right|^{2-n_{T}}\left(\frac{k}{k_{\max }}\right)^{n_{T}}$, where we defined, for the sake of conciseness, $k_{\max }=a_{1} H_{1}$. The results reported so far for the power spectra and for the relevant spectral indices refer to the case of the canonical action of Eq. (2.1). In the case of conformally related actions (such as the ones discussed in Refs. [51]) the relevant results can be found in Appendix C.

\section{B. The power spectra after reentry}

Terrestrial interferometers and space-borne detectors operate at the present time and will necessarily measure the cosmic graviton spectrum for typical wavelengths shorter than the Hubble radius. While the largest wavelengths of the problem (i.e., smallest $k$ modes) reentered after matter-radiation equality, the shortest wavelengths (i.e., largest $k$ modes) crossed the effective horizon at different epochs after the end of the inflationary stage of expansion and even before the onset of the radiationdominated phase.

The reentry depends on the post-inflationary thermal history and on the expansion rate that can be very different from the one of a radiation-dominated plasma. When the refractive index is not dynamical the previous observation leads to a characteristic class of violet spectral energy distribution $[26,28,50]$ and it will be interesting to see what happens in the present situation. Provided the reentry occurs when

$$
k^{2}=\left|\frac{\ddot{b}_{\text {re }}}{b_{\text {re }}}\right|, \quad \ddot{b}_{\text {re }} \neq 0,
$$

then $k \eta_{\text {re }}=\mathcal{O}(1)$. However, as already remarked above [see Eq. (3.2)], if $\ddot{b}_{\mathrm{re}} \rightarrow 0$ in the vicinity of the turning point, then $k \eta_{\mathrm{re}} \ll 1$. For $\eta \geq \eta_{\mathrm{re}}$, the solution of Eq. (2.20) can be expressed as

$$
\begin{aligned}
f_{k}(\eta) & =c_{+}(k) \bar{f}_{\mathrm{re}}(\eta)+c_{-}(k) \bar{f}_{\mathrm{re}}^{*}(\eta), \\
g_{k}(\eta) & =c_{+}(k) \bar{g}_{\mathrm{re}}(\eta)+c_{-}(k) \bar{g}_{\mathrm{re}}^{*}(\eta),
\end{aligned}
$$

where $\bar{f}_{\text {re }}(\eta)$ and $\bar{g}_{\text {re }}(\eta)$ are the mode functions inside the effective horizon (i.e., quantum mechanically normalized plane waves in the crudest approximation). From the continuity of $f_{k}(\eta)$ and $g_{k}(\eta)$, Eqs. (3.3) and (3.4) imply

$$
f_{k}\left(\eta_{\mathrm{re}}\right)=\bar{f}_{\mathrm{ex}}\left(\frac{b_{\mathrm{re}}}{b_{\mathrm{ex}}}\right)+b_{\mathrm{re}} b_{\mathrm{ex}}\left(\bar{g}_{\mathrm{ex}}-\mathcal{F}_{\mathrm{ex}} \bar{f}_{\mathrm{ex}}\right) \mathcal{J}\left(\eta_{\mathrm{ex}}, \eta_{\mathrm{re}}\right),
$$

$$
\begin{gathered}
g_{k}\left(\eta_{\mathrm{re}}\right)=\frac{b_{\mathrm{ex}}}{b_{\mathrm{re}}} \bar{g}_{\mathrm{ex}}+\bar{f}_{\mathrm{ex}}\left[\left(\frac{b_{\mathrm{re}}}{b_{\mathrm{ex}}}\right) \mathcal{F}_{\mathrm{re}}-\left(\frac{b_{\mathrm{ex}}}{b_{\mathrm{re}}}\right) \mathcal{F}_{\mathrm{ex}}\right] \\
+b_{\mathrm{re}} b_{\mathrm{ex}} \mathcal{F}_{\mathrm{re}}\left(\bar{g}_{\mathrm{ex}}-\mathcal{F}_{\mathrm{ex}} \bar{f}_{\mathrm{ex}}\right) \mathcal{J}\left(\eta_{\mathrm{ex}}, \eta_{\mathrm{re}}\right), \\
\mathcal{J}\left(\eta_{\mathrm{ex}}, \eta_{\mathrm{re}}\right)=\int_{\eta_{\mathrm{ex}}}^{\eta_{\mathrm{re}}} \frac{d \eta}{b^{2}(\eta)} .
\end{gathered}
$$


By continuity, Eq. (3.16), evaluated at $\eta_{\mathrm{re}}$, must coincide with Eqs. (3.17) and (3.18); thus, $c_{ \pm}(k)$ can be determined after some simple algebraic manipulation?

$$
\begin{aligned}
c_{+}(k)= & (-i)\left\{\left[\bar{g}_{\mathrm{re}}^{*} \bar{f}_{\mathrm{ex}}\left(\frac{b_{\mathrm{re}}}{b_{\mathrm{ex}}}\right)-\bar{f}_{\mathrm{re}}^{*} \bar{g}_{\mathrm{ex}}\left(\frac{b_{\mathrm{ex}}}{b_{\mathrm{re}}}\right)\right]\right. \\
& -\bar{f}_{\mathrm{re}}^{*} \bar{f}_{\mathrm{ex}}\left[\mathcal{F}_{\mathrm{re}}\left(\frac{b_{\mathrm{re}}}{b_{\mathrm{ex}}}\right)-\mathcal{F}_{\mathrm{ex}}\left(\frac{b_{\mathrm{ex}}}{b_{\mathrm{re}}}\right)\right] \\
& \left.+b_{\mathrm{re}} b_{\mathrm{ex}}\left(\bar{g}_{\mathrm{ex}}-\mathcal{F}_{\mathrm{ex}} \bar{f}_{\mathrm{ex}}\right)\left(\bar{g}_{\mathrm{re}}^{*}-\mathcal{F}_{\mathrm{re}} \bar{f}_{\mathrm{re}}^{*}\right) \mathcal{J}\left(\eta_{\mathrm{ex}}, \eta_{\mathrm{re}}\right)\right\},
\end{aligned}
$$

$$
\begin{aligned}
c_{-}(k)= & i\left\{\left[\bar{g}_{\mathrm{re}} \bar{f}_{\mathrm{ex}}\left(\frac{b_{\mathrm{re}}}{b_{\mathrm{ex}}}\right)-\bar{f}_{\mathrm{re}} \bar{g}_{\mathrm{ex}}\left(\frac{b_{\mathrm{ex}}}{b_{\mathrm{re}}}\right)\right]\right. \\
& -\bar{f}_{\mathrm{re}} \bar{f}_{\mathrm{ex}}\left[\mathcal{F}_{\mathrm{re}}\left(\frac{b_{\mathrm{re}}}{b_{\mathrm{ex}}}\right)-\mathcal{F}_{\mathrm{ex}}\left(\frac{b_{\mathrm{ex}}}{b_{\mathrm{re}}}\right)\right] \\
& \left.+b_{\mathrm{re}} b_{\mathrm{ex}}\left(\bar{g}_{\mathrm{ex}}-\mathcal{F}_{\mathrm{ex}} \bar{f}_{\mathrm{ex}}\right)\left(\bar{g}_{\mathrm{re}}-\mathcal{F}_{\mathrm{re}} \bar{f}_{\mathrm{re}}\right) \mathcal{J}\left(\eta_{\mathrm{ex}}, \eta_{\mathrm{re}}\right)\right\} .
\end{aligned}
$$

Inside the Hubble radius, the mode functions are plane waves or, more precisely, the limit of Hankel functions for large values of their arguments [47,48]. We can then express directly Eqs. (3.20) and (3.21) by using the plane wave limit of the corresponding mode functions:

$$
\begin{aligned}
c_{+}(k)= & \frac{e^{-i k\left(\eta_{\mathrm{re}}-\eta_{\mathrm{ex}}\right)}}{2 i k}\left[\frac{b_{\mathrm{re}}}{b_{\mathrm{ex}}}\left(i k-\mathcal{F}_{\mathrm{re}}\right)+\frac{b_{\mathrm{ex}}}{b_{\mathrm{re}}}\left(i k+\mathcal{F}_{\mathrm{ex}}\right)\right. \\
& \left.+b_{\mathrm{re}} b_{\mathrm{ex}}\left(\mathcal{F}_{\mathrm{re}}-i k\right)\left(\mathcal{F}_{\mathrm{ex}}+i k\right) \mathcal{J}\left(\eta_{\mathrm{ex}}, \eta_{\mathrm{re}}\right)\right], \\
c_{-}(k)= & \frac{e^{-i k\left(\eta_{\mathrm{re}}+\eta_{\mathrm{ex}}\right)}}{2 i k}\left[\frac{b_{\mathrm{re}}}{b_{\mathrm{ex}}}\left(i k+\mathcal{F}_{\mathrm{re}}\right)-\frac{b_{\mathrm{ex}}}{b_{\mathrm{re}}}\left(i k+\mathcal{F}_{\mathrm{ex}}\right)\right. \\
& \left.-b_{\mathrm{re}} b_{\mathrm{ex}}\left(\mathcal{F}_{\mathrm{re}}+i k\right)\left(\mathcal{F}_{\mathrm{ex}}+i k\right) \mathcal{J}\left(\eta_{\mathrm{ex}}, \eta_{\mathrm{re}}\right)\right] .
\end{aligned}
$$

Since the coefficients $c_{ \pm}(k)$ satisfy $\left|c_{+}(k)\right|^{2}-\left|c_{-}\right|^{2}=1$, it is sufficient to determine just one of the two square moduli. If the exit occurs for $\eta<-\eta_{*}$ and the reentry takes place when the refractive index is not dynamical, Eqs. (3.22) and (3.23) can be written more explicitly

\footnotetext{
${ }^{9}$ For the derivation of Eqs. (3.20) and (3.21), it is useful to recall that $\bar{f}_{\text {re }} \bar{g}_{\text {re }}^{*}-\bar{f}_{\text {re }}^{*} \bar{g}_{\text {re }}=i$, as implied by the constancy of the Wronskian when the second-order differential equation for $f_{k}$ is written in the form (2.20); the constancy of the Wronskian also implies for $\eta>\eta_{\text {re }}$ that the coefficients $c_{ \pm}(k)$ must satisfy $\left|c_{+}(k)\right|^{2}-\left|c_{-}\right|^{2}=1$.
}

$$
\begin{aligned}
c_{+}(k)= & \frac{e^{-i k\left(\tau_{\mathrm{re}}-\eta_{\mathrm{ex}}\right)}}{2 i k}\left[\frac{a_{\mathrm{re}}}{b_{\mathrm{ex}}}\left(i k-\mathcal{H}_{\mathrm{re}}\right)+\frac{b_{\mathrm{ex}}}{a_{\mathrm{re}}}\left(i k+\mathcal{F}_{\mathrm{ex}}\right)\right. \\
& \left.+a_{\mathrm{re}} b_{\mathrm{ex}}\left(\mathcal{H}_{\mathrm{re}}-i k\right)\left(\mathcal{F}_{\mathrm{ex}}+i k\right) \mathcal{J}\left(\eta_{\mathrm{ex}}, \tau_{\mathrm{re}}\right)\right], \\
c_{-}(k)= & \frac{e^{-i k\left(\tau_{\mathrm{re}}+\eta_{\mathrm{ex}}\right)}}{2 i k}\left[\frac{a_{\mathrm{re}}}{b_{\mathrm{ex}}}\left(i k+\mathcal{H}_{\mathrm{re}}\right)-\frac{b_{\mathrm{ex}}}{b_{\mathrm{re}}}\left(i k+\mathcal{F}_{\mathrm{ex}}\right)\right. \\
& \left.-a_{\mathrm{re}} b_{\mathrm{ex}}\left(\mathcal{H}_{\mathrm{re}}+i k\right)\left(\mathcal{F}_{\mathrm{ex}}+i k\right) \mathcal{J}\left(\eta_{\mathrm{ex}}, \tau_{\mathrm{re}}\right)\right],
\end{aligned}
$$

where this time

$$
\mathcal{J}\left(\eta_{\mathrm{ex}}, \tau_{\mathrm{re}}\right)=\int_{\eta_{\mathrm{ex}}}^{\eta_{*}} \frac{d \eta}{b^{2}(\eta)}+\int_{\tau_{*}}^{\tau_{\mathrm{re}}} \frac{d \tau}{a^{2}(\tau)}
$$

Because $b(\eta)$ always increases throughout the refractive phase and even later [see Eq. (2.31) and discussion therein], in Eqs. (3.22) and (3.23) the terms proportional to $\left|b_{\mathrm{ex}} / b_{\mathrm{re}}\right|$ can be neglected in comparison with $\left|b_{\mathrm{re}} / b_{\mathrm{ex}}\right|$. Following this logic, the approximate form of $\left|c_{-}(k)\right|^{2}$ becomes

$$
\begin{aligned}
\left|c_{-}(k)\right|^{2} \simeq & \frac{1}{4}\left(\frac{b_{\mathrm{re}}}{b_{\mathrm{ex}}}\right)^{2}\left(1+\frac{\mathcal{F}_{\mathrm{re}}^{2}}{k^{2}}\right) \\
& \times\left[1-2 \mathcal{F}_{\mathrm{ex}} b_{\mathrm{ex}}^{2} \mathcal{J}+b_{\mathrm{ex}}^{4}\left(\mathcal{F}_{\mathrm{ex}}^{2}+k^{2}\right) \mathcal{J}^{2}\right] .
\end{aligned}
$$

Equation (3.27) allows for a swift determination of the power spectrum and of the spectral energy distribution in the limit $k \tau \gg 1$, i.e., when the relevant wavelengths are all inside the Hubble radius:

$$
\begin{gathered}
\mathcal{P}(k, \tau)=\frac{4 k^{2}}{\pi^{2} \bar{M}_{P}^{2}}\left|c_{-}(k)\right|^{2}\left[1+\mathcal{O}\left(\frac{1}{k^{2} \tau^{2}}\right)\right], \\
\Omega_{g w}(k, \tau)=\frac{k^{4}}{3 H^{2} \bar{M}_{P}^{2} \pi^{2} a^{4}}\left|c_{-}(k)\right|^{2}\left[1+\mathcal{O}\left(\frac{1}{k^{2} \tau^{2}}\right)\right] .
\end{gathered}
$$

By taking the ratio between Eqs. (3.28) and (3.29) we recover the standard relation between the power spectrum and the spectral energy density valid when the relevant wavelengths are shorter than the Hubble radius at a given epoch:

$$
\Omega_{g w}(k, \tau)=\frac{k^{2}}{12 a^{2} H^{2}} \mathcal{P}_{T}(k, \tau)\left[1+\mathcal{O}\left(\frac{1}{k^{2} \tau^{2}}\right)\right] .
$$

Consequently, inside the Hubble radius we can evaluate indifferently either the power spectrum or the spectral energy distribution. Equation (3.30) holds when all the modes are inside the effective horizon and also depends on the specific thermal history. Different thermal histories lead to a different power spectrum and to a different spectral energy distribution. To determine the spectral energy distribution we shall therefore consider different thermal histories and the evolutions of the associated effective horizons. 


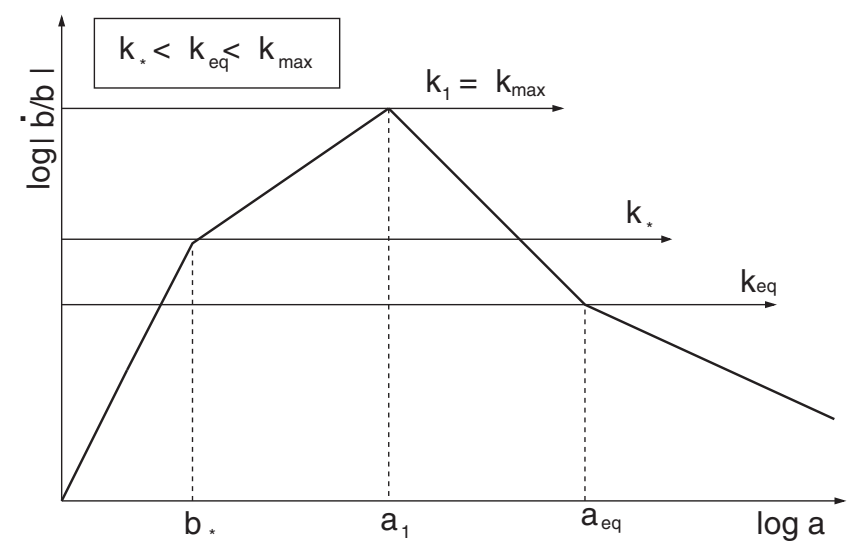

FIG. 1. We schematically illustrate the evolution of the effective horizon in the minimal situation where the radiation background suddenly dominates after inflation.

\section{Different thermal histories}

The different thermal histories and their effects on the spectral energy distribution can be understood by drawing the effective horizon in various physical situations. In Fig. 1, on the vertical axis, we plot the common logarithm of $\mathcal{F}=$ $\dot{b} / b$ and we simultaneously compare it with the wave numbers of the problem. ${ }^{10}$ In practice the conditions $k \eta_{\mathrm{ex}}=$ $\mathcal{O}(1)$ and $k \tau_{\text {re }}=\mathcal{O}(1)$ will always be verified except that in the case of a reentry during radiation when $k \tau_{\text {re }} \ll 1$ and $a_{\mathrm{re}}^{\prime \prime}=0$. According to Fig. 1 we have three different classes of modes: (i) the modes exiting the effective horizon during the refractive phase and reentering after equality (i.e., $k<a_{\mathrm{eq}} H_{\mathrm{eq}}$ ), (ii) the modes exiting the effective horizon during the refractive phase and reentering during radiation (i.e., $a_{\mathrm{eq}} H_{\mathrm{eq}}<k<a_{*} H_{*}$ ), and (iii) the modes exiting the effective horizon after the end of the refractive phase and reentering during radiation (i.e., $a_{*} H_{*}<k<a_{1} H_{1}$ ). The different regions are separated in Fig. 1 by three horizontal arrows and the typical wave numbers (i.e., $k_{1}, k_{*}$, and $k_{\text {eq }}$ ) define the three branches of the spectral energy density (or of the power spectrum). Either the inflationary phase continues after $b_{*}$ or the radiation-dominated epoch suddenly kicks in. Between these two possibilities, the former is more generic than the latter which would correspond, in the notation of Fig. 1, to the limit $b_{*} \rightarrow a_{1}$; this is why, in Fig. 1, we preferred to distinguish clearly the two scales by assuming $b_{*} \ll a_{1}$. The three different branches of the spectral energy distribution illustrated in Fig. 1 can be deduced from Eqs. (3.27)-(3.29); the result of this explicit computation is

$$
\begin{aligned}
\Omega_{g w}\left(k, \tau_{\mathrm{re}}\right) \simeq & \left(\frac{H_{1}}{M_{P}}\right)^{2} \mathcal{B}\left(b_{*}, n_{*}, \epsilon, n_{T}\right)\left(\frac{k}{a_{*} H_{*}}\right)^{-2 \epsilon /(1-\epsilon)}, \\
& a_{*} H_{*}<k \leq a_{1} H_{1},
\end{aligned}
$$

\footnotetext{
${ }^{10}$ This comparison is physically motivated since the crossing condition can also be written as: $k^{2}=\mathcal{F}^{2}+\dot{\mathcal{F}}$.
}

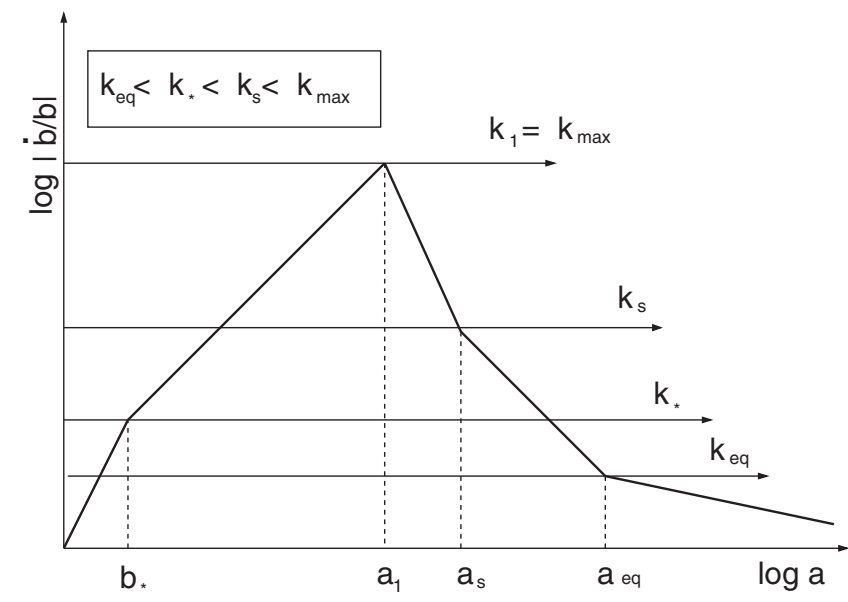

FIG. 2. We schematically illustrate the evolution of the effective horizon in the case where the dominance of radiation is delayed by the presence of a stiff phase.

$$
\begin{aligned}
\Omega_{g w}\left(k, \tau_{\mathrm{re}}\right) \simeq & \left(\frac{H_{1}}{M_{P}}\right)^{2} \mathcal{B}\left(b_{*}, n_{*}, \epsilon, n_{T}\right)\left(\frac{k}{a_{*} H_{*}}\right)^{n_{T}}, \\
& a_{\mathrm{eq}} H_{\mathrm{eq}}<k \leq a_{*} H_{*}, \\
\Omega_{g w}\left(k, \tau_{\mathrm{re}}\right) \simeq & \left(\frac{H_{1}}{M_{P}}\right)^{2} \mathcal{B}\left(b_{*}, n_{*}, \epsilon, n_{T}\right)\left(\frac{k}{a_{*} H_{*}}\right)^{n_{T}} \\
& \times\left(\frac{k}{a_{\mathrm{eq}} H_{\mathrm{eq}}}\right)^{-2}, \quad k \leq a_{\mathrm{eq}} H_{\mathrm{eq}},
\end{aligned}
$$

where $\mathcal{B}\left(b_{*}, n_{*}, \epsilon, n_{T}\right)$ is given by ${ }^{11}$

$\mathcal{B}\left(b_{*}, n_{*}, \epsilon, n_{T}\right)=\frac{2}{3 \pi}\left(\frac{\Omega_{R 0}}{\Omega_{M 0}}\right) n_{*}^{3-n_{T}}\left|1+\frac{\alpha}{1-\epsilon}\right|^{2-n_{T}}$.

The spectral index $n_{T}$ appearing in Eqs. (3.31)-(3.34) is instead:

$n_{T}=\frac{3 \alpha-2 \epsilon}{(1+\alpha-\epsilon)}=\frac{3 \alpha}{1+\alpha}+\frac{(\alpha-2) \epsilon}{(1+\alpha)^{2}}+\mathcal{O}\left(\epsilon^{2}\right)$

where the second equality follows in the limit $\epsilon \ll 1$. As it must, the exact expression of Eq. (3.35) coincides with Eq. (3.12). The quasiflat branch of Eq. (3.31) is caused by the modes that exited the effective horizon for $a>b_{*}$ and reentered during the radiation-dominated epoch (i.e., for $\left.a>a_{1}\right)$. The second branch of the spectrum, reported in Eq. (3.32), involves the modes that exited the effective horizon during the refractive phase and reentered all along the radiation stage. Finally, the standard infrared branch

\footnotetext{
${ }^{11}$ Note that $\Omega_{M 0}$ and $\Omega_{R 0}$ denote throughout the present values of the critical fractions of matter and radiation in the concordance paradigm. Both $\mathcal{B}\left(b_{*}, n_{*}, \epsilon, n_{T}\right)$ and $n_{T}$ are affected by the different forms of the graviton action. While the following results come directly from Eq. (2.1), the modifications induced by a different form of the original action can be found in Appendix C.
} 
corresponds to modes that are exiting the effective horizon during the refractive epoch and reentering during the matterdominated phase (i.e., $k<k_{\text {eq }}$ in the terminology of Fig. 1). There are no compelling reasons why the physical situation illustrated by Fig. 1 should be considered preferable to some other potentially viable evolution of the effective horizon. Different thermal histories can be envisaged and cannot be ruled out by the present version of the concordance scenario. Prior to nucleosynthesis, there are no direct tests of the thermodynamical state of the Universe and, therefore, the effective equation of state of the primeval plasma can be arbitrarily different than the one of radiation. In Fig. 2 the effective horizon is illustrated in the case where the postinflationary expansion rate is slower than the one of a radiation-dominated plasma; in this case between $a_{1}$ and $a_{s}$ we have

$$
\frac{\dot{b}}{b}=\mathcal{H}=a H \propto a^{-(3 w+1) / 2}, \quad w>\frac{1}{3},
$$

where $w$ denotes the barotropic index of the stiff postinflationary phase. Note, for comparison, that $\mathcal{H} \propto a$ during inflation while, in the radiation stage, $\mathcal{H} \propto 1 / a$ (see also Fig. 1). In the case of a stiff post-inflationary phase we have instead that (at most) $\mathcal{H} \propto a^{-2}$, as implied by Eq. (3.36) for $w \rightarrow 1$.

The evolution sketched in Fig. 2 leads to a spectral energy distribution characterized by four different branches: the wave numbers $k<a_{\mathrm{eq}} H_{\mathrm{eq}}$ correspond to scales hitting the effective horizon the first time during the refractive phase and reentering after matter-radiation equality: their spectral energy distribution will then have the same slope as Eq. (3.33). Following the same way of reasoning $\Omega_{g w} \propto\left|k \tau_{*}\right|^{n_{T}}$ whenever $a_{\mathrm{eq}} H_{\mathrm{eq}}<k<a_{*} H_{*}$ : in Fig. 2 this part of the spectrum corresponds to those modes exiting during the refractive phase and reentering during the radiation epoch. The supplementary branch of the spectrum implied by Fig. 2 is caused by those modes exiting in the course of the inflationary phase and reentering during the stiff phase: in this branch the spectral energy density scales as $\Omega_{g w} \propto\left|k \tau_{s}\right|^{m_{T}}$ where the spectral index $m_{T}$ is now given by

$$
m_{T}=4-\frac{2}{1-\epsilon}-\frac{4}{3 w+1} .
$$

If the expansion rate is slower than radiation, the slope in this branch can be very steep (i.e., even violet) with $m_{T}=$ $\mathcal{O}(1)$ in the limit $w \rightarrow 1$. Incidentally if the expansion rate is faster than radiation ${ }^{12}$ it can happen that $m_{T}<0$.

While the cases illustrated by Figs. 1 and 2 are the most promising from the viewpoint of the potential signals (as we shall see in the following section), there are other

\footnotetext{
${ }^{12}$ For instance in the case $w \rightarrow 0$ we would have $m_{T} \rightarrow-2$.
}

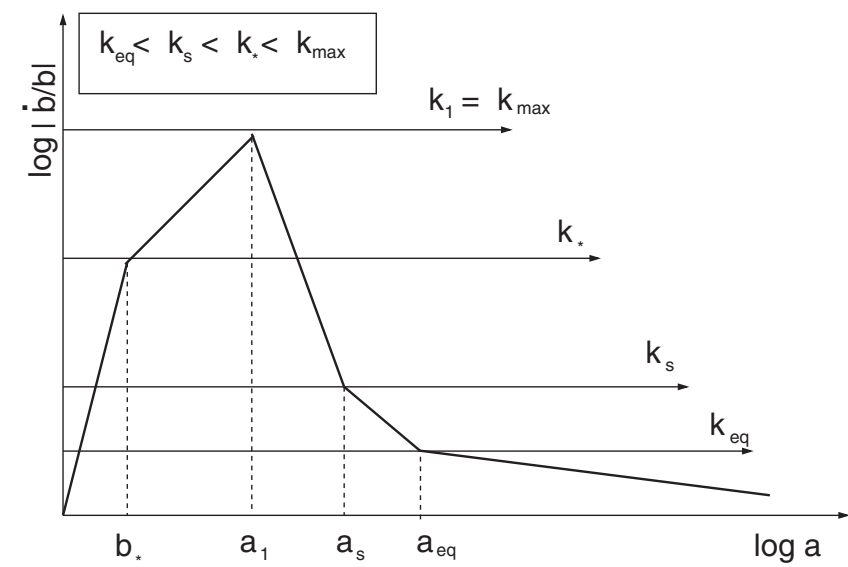

FIG. 3. The dominance of radiation is delayed but the refractive and the stiff phases are both longer than in Fig. 2.

possible evolutions of the effective horizon where the resulting spectral energy distribution does not have a flat (or decreasing) plateau and it always increases. In this connection, Fig. 3 illustrates a possibility complementary to the one of Fig. 2 but leading to a different spectrum. Both in Figs. 2 and 3 a stiff phase precedes the ordinary radiation epoch. However, the refractive and the stiff phases of Fig. 3 are longer then in Fig. 2. This occurrence implies the possibility of modes exiting the effective horizon during the refractive phase and reentering during the stiff phase. This different dynamical situation implies that the intermediate branch of the spectrum (i.e., $a_{*} H_{*}<k<a_{s} H_{s}$ ) is not quasiflat anymore. Furthermore, using Eqs. (3.27) and (3.29), $\Omega_{g w}$ scales as $\left|k \tau_{s}\right|^{s_{T}}$ where now $s_{T}$ is given by

$s_{T}=2-\frac{4}{3 w+1}+n_{T} \simeq 2-\frac{4}{3 w+1}+\frac{3 \alpha}{1+\alpha}+\mathcal{O}(\epsilon)$.

For instance, for $w=1$ we will have that $s_{T}=1+\alpha$ while $m_{T}=\mathcal{O}(1)$. The slope of Eq. (3.38) is always increasing and since also the other branches of the spectral energy density are increasing (thought at a different rate), all the energy of this spectrum will be concentrated in the highest frequency regime and this is the reason why the detectability prospects are, in this situation, less promising than in the case of a sufficiently long plateau at high frequency. Various other examples can be analyzed by using the approximate methods described in this section but they are not central to the present discussion. We remark, once more, that the results reported here refer to the action (2.1). There can, however, be conformally related parametrizations of the action for the relic gravitons (see Appendix A). In this case a generalized action can be written depending on a parameter $\gamma$ (see Appendix B). The results of this section are clearly affected by the value of $\gamma$ and some discussions of this point can be found in Appendix C.

The results of this section can be summarized, in short, as follows. If the radiation background suddenly follows the 
inflationary phase, the spectral energy density has overall three different branches. If the thermal history is different there might be either one or two further branches in the spectrum. Generically, the presence of the refractive index induces a growing spectral energy distribution at intermediate frequencies and a quasiflat plateau at high frequencies. In the intermediate branch the spectral index is directly related to the rate of evolution of the refractive index during inflation [i.e., $\alpha$, see Eq. (3.35)]. If the radiation-dominated epoch does not immediately follow the inflationary phase, the high-frequency region is not quasiflat and the slope does also depend on the barotropic index of the post-inflationary epoch [see Eqs. (3.37) and (3.38)]. It is useful to recall that this situation is radically different from the one of conventional inflationary models where the intermediate branch of the spectrum is quasiflat. Conversely, in the present case, the spectral energy distribution is quasiflat at high frequencies and increasing over the intermediate frequencies. In the following sections, the different branches of the spectral energy distributions and the typical frequencies will be explicitly discussed.

\section{DETECTABILITY PROSPECTS}

\section{A. Basic considerations}

The phenomenological signatures of the relic gravitons are customarily assessed in terms of the comoving frequency that is defined as $\nu=k /(2 \pi)$, where $k$ denotes the comoving wave number. Four complementary quantities describe the cosmic graviton background: (a) the tensor power spectrum [denoted by $\mathcal{P}_{T}\left(\nu, \tau_{0}\right)$ ], (b) the spectral energy distribution [i.e., $\Omega_{g w}\left(\nu, \tau_{0}\right)$ ], (c) the chirp amplitude $h_{c}\left(\nu, \tau_{0}\right)$, and (d) the spectral amplitude $S_{h}\left(\nu, \tau_{0}\right)$ (measured in units of $\left.\mathrm{Hz}^{-1}=\mathrm{sec}\right)$. Except for $S_{h}\left(\nu, \tau_{0}\right)$ the three remaining variables are dimensionless. The chirp amplitude is, by definition, $h_{c}^{2}\left(\nu, \tau_{0}\right)=\mathcal{P}_{T}\left(\nu, \tau_{0}\right) / 2$. The tensor power spectrum at the present time is related to the spectral energy distribution as

$$
\Omega_{g w}\left(\nu, \tau_{0}\right)=\frac{3 \pi^{2} \nu^{2}}{4 H_{0}^{2} a_{0}^{2}} \mathcal{P}_{T}\left(\nu, \tau_{0}\right) .
$$

Equation (4.1) as well as all other equations in this section involve wavelengths shorter than the Hubble radius at the present time $\tau_{0}$. The chirp amplitude and the spectral amplitude are directly related to $\Omega_{g w}\left(\nu, \tau_{0}\right)$ in the following manner:

$$
\begin{aligned}
& h_{c}\left(\nu, \tau_{0}\right)=\frac{1}{\pi} \sqrt{\frac{3}{2}}\left(\frac{H_{0} a_{0}}{\nu}\right) \sqrt{\Omega_{g w}\left(\nu, \tau_{0}\right)}, \\
& \mathcal{S}_{h}\left(\nu, \tau_{0}\right)=\frac{3 H_{0}^{2} a_{0}^{2}}{4 \pi^{2} \nu^{3}} \Omega_{g w}\left(\nu, \tau_{0}\right) .
\end{aligned}
$$

Equation (4.2) implies that $h_{c}^{2}\left(\nu, \tau_{0}\right)=2 \nu \mathcal{S}_{h}\left(\nu, \tau_{0}\right)$ and since the detectors of gravitational radiation are operating in the audio band (i.e., between a few $\mathrm{Hz}$ and $10 \mathrm{kHz}$ ) it is useful to recall the explicit relations between the various quantities mentioned above for typical frequencies $\nu=\mathcal{O}(100) \mathrm{Hz}$ where the sensitivities of wide-band detectors to cosmic graviton background are (approximately) $\operatorname{maximal}^{13}$ :

$$
\begin{aligned}
& h_{c}\left(\nu, \tau_{0}\right)=1.263 \times 10^{-20}\left(\frac{100 \mathrm{~Hz}}{\nu}\right) \sqrt{h_{0}^{2} \Omega_{g w}\left(\nu, \tau_{0}\right)}, \\
& \mathcal{P}_{T}\left(\nu, \tau_{0}\right)=3.190 \times 10^{-40}\left(\frac{100 \mathrm{~Hz}}{\nu}\right)^{2} h_{0}^{2} \Omega_{g w}\left(\nu, \tau_{0}\right), \\
& \mathcal{S}_{h}\left(\nu, \tau_{0}\right)=7.981 \times 10^{-43}\left(\frac{100 \mathrm{~Hz}}{\nu}\right)^{3} h_{0}^{2} \Omega_{g w}\left(\nu, \tau_{0}\right) \mathrm{Hz}^{-1} .
\end{aligned}
$$

From Eqs. (4.3)-(4.5) the orders of magnitude of the different variables employed in the description of relic graviton backgrounds can be explicitly assessed.

\section{B. Pivotal frequencies}

The spectral energy distribution is characterized by various typical frequencies which are determined from the wave numbers appearing in Figs. 1-3. The smallest frequency range of the spectrum follows from the pivot wave number $k_{p}$ at which the scalar and tensor power spectra are assigned [16-19]:

$$
\nu_{p}=\frac{k_{p}}{2 \pi}=3.092 \times 10^{-18} \mathrm{~Hz}=3.092 \mathrm{aHz} .
$$

The frequency associated with the dominance of dark energy is of the same order of Eq. (4.6) and it is fixed by $\Omega_{M 0}$ and $\Omega_{\Lambda}$; in the case of the concordance paradigm we have

$\nu_{\Lambda}=1.638\left(\frac{h_{0}}{0.719}\right)\left(\frac{\Omega_{\mathrm{M} 0}}{0.258}\right)^{1 / 3}\left(\frac{\Omega_{\Lambda}}{0.742}\right)^{-1 / 3} \mathrm{aHz}$.

Since the equality wave number is $k_{\text {eq }}=0.0732\left[h_{0}^{2} \Omega_{R 0} /\right.$ $\left.\left(4.15 \times 10^{-5}\right)\right]^{-1 / 2} h_{0}^{2} \Omega_{M 0} \mathrm{Mpc}^{-1}$ the related frequency $\nu_{\text {eq }}$ is

$\nu_{\mathrm{eq}}=1.317 \times 10^{-17}\left(\frac{h_{0}^{2} \Omega_{\mathrm{M} 0}}{0.1364}\right)\left(\frac{h_{0}^{2} \Omega_{\mathrm{R} 0}}{4.15 \times 10^{-5}}\right)^{-1 / 2} \mathrm{~Hz}$.

The frequency $\nu_{b b n}=\mathcal{O}\left(10^{-2}\right) \mathrm{nHz}$ enters the big-bang nucleosynthesis constraint directly [see below Eq. (4.13)]

\footnotetext{
${ }^{13}$ Since $\Omega_{g w}$ contains the inverse of $\rho_{\text {crit }}, h_{0}^{2} \Omega_{g w}$ is in fact independent on $h_{0}$.
} 
and sets the scale for the suppression of the cosmic graviton background due to neutrino free streaming $[12,13]$. The explicit expression of the big-bang nucleosynthesis frequency is ${ }^{14}$

$$
\begin{aligned}
\nu_{b b n}= & 2.252 \times 10^{-11}\left(\frac{g_{\rho}}{10.75}\right)^{1 / 4}\left(\frac{T_{b b n}}{\mathrm{MeV}}\right) \\
& \times\left(\frac{h_{0}^{2} \Omega_{\mathrm{R} 0}}{4.15 \times 10^{-5}}\right)^{1 / 4} \mathrm{~Hz} .
\end{aligned}
$$

The presence of the refractive phase illustrated in Figs. 1 and 2 introduces two further frequencies:

$$
\begin{gathered}
\nu_{*}=p\left(\alpha, \epsilon, N_{*}, N_{t}\right) \nu_{\max }, \\
p\left(\alpha, \epsilon, N_{*}, N_{t}\right)=\left|1+\frac{\alpha}{1-\epsilon}\right| e^{N_{*}(\alpha+1)-N_{t}}, \\
\nu_{\max }=1.95 \times 10^{8}\left(\frac{\epsilon}{0.001}\right)^{1 / 4}\left(\frac{\mathcal{A}_{\mathcal{R}}}{2.41 \times 10^{-9}}\right)^{1 / 4} \\
\times\left(\frac{h_{0}^{2} \Omega_{R 0}}{4.15 \times 10^{-5}}\right)^{1 / 4} \mathrm{~Hz}
\end{gathered}
$$

where $\mathcal{A}_{\mathcal{R}}$ denotes the amplitude of the power spectrum of curvature inhomogeneities at the wave number $k_{p}$. Even if Eq. (4.11) suggests that $\nu_{\max }=\mathcal{O}(200) \mathrm{MHz}$, the value of the end-point frequency of the spectrum may exceed $\nu_{\max }$ since it depends on the post-inflationary thermal history [32]. For the thermal histories of Figs. 2 and 3, the spectral energy distribution may extend up to $\nu_{\text {spike }}=\nu_{\max } / \sigma>$ $\nu_{\max }$ (with $\sigma<1$ ). While a similar spike (with different physical features) may also arise when the refractive index is not dynamical [32], in this particular case two new frequencies appear and they are defined as

$$
\begin{aligned}
\nu_{s} & =\sigma^{3(w+1) /(3 w-1)} \nu_{\max }, \quad \nu_{\text {spike }}=\nu_{\max } / \sigma, \\
\sigma & =\left(\frac{H_{\max }}{H_{r}}\right)^{\frac{1-3-3 w}{6(w+1)}}
\end{aligned}
$$

where $H_{r}$ denotes the Hubble rate at the onset of the radiation dominance, i.e., right after the stiff phase. The difference between $\nu_{\max }$ and $\nu_{\text {spike }}$ comes essentially from the redshift during the stiff stage of expansion.

\section{Phenomenological constraints}

In the low-frequency range the tensor to scalar ratio of Eq. (3.14) is bounded from above not to conflict with the observed temperature and polarization anisotropies of the $\mathrm{CMB}$; in the present analysis we specifically required

\footnotetext{
${ }^{14}$ Note that $g_{\rho}$ denotes the effective number of relativistic d.o.f. entering the total energy density of the plasma and $T_{b b n}$ is the putative temperature of big-bang nucleosynthesis.
}

$r_{T}\left(\nu_{p}\right)<0.06$, as it follows from a joint analysis of Planck and BICEP2/Keck array data [16]. As already mentioned in the Introduction, slightly less restrictive bounds are often used in the current literature and they amount to demanding $r_{T}\left(\nu_{p}\right)<\mathcal{O}(0.1)$ [18,19,23]. The pulsar timing measurements impose instead the limit $\Omega_{g w}\left(\nu_{\text {pulsar }}, \tau_{0}\right)<1.9 \times 10^{-8}$ at the frequency $\nu_{\text {pulsar }}=$ $\mathcal{O}(10) \mathrm{nHz}$ corresponding to the inverse of the observation time along which the pulsars timing has been monitored [52-57]. The big-bang nucleosynthesis sets an indirect constraint on the extra-relativistic species (and, among others, on the relic gravitons) at the time when light nuclei have been formed [58-60]. This limit is often expressed in terms of $\Delta N_{\nu}$ representing the contribution of supplementary (massless) neutrino species (see, e.g., [61]) but the extra-relativistic species do not need to be fermionic. If, as in our case, the additional species are relic gravitons we will have to demand that

$$
h_{0}^{2} \int_{\nu_{b b n}}^{\nu_{\max }} \Omega_{g w}\left(\nu, \tau_{0}\right) d \ln \nu=5.61 \times 10^{-6} \Delta N_{\nu}\left(\frac{h_{0}^{2} \Omega_{\gamma 0}}{2.47 \times 10^{-5}}\right) .
$$

The bounds on $\Delta N_{\nu}$ range from $\Delta N_{\nu} \leq 0.2$ to $\Delta N_{\nu} \leq 1$ so that the right-hand side of Eq. (4.13) turns out to be between $10^{-6}$ and $10^{-5}$. The basic considerations discussed here can be complemented by other bounds which are, however, less constraining than the ones mentioned above. The same logic employed for the derivation of Eq. (4.13) can be applied at the decoupling of matter and radiation. While the typical frequency of big-bang nucleosynthesis is $\mathcal{O}\left(10^{-10}\right) \mathrm{Hz}$ the typical frequencies of matter-radiation equality is $\mathcal{O}\left(10^{-16}\right) \mathrm{Hz}$ [see Eqs. (4.8) and (4.9)]. Since the decoupling between matter and radiation occurs after equality we have that

$$
h_{0}^{2} \int_{\nu_{\mathrm{dec}}}^{\nu_{\max }} \Omega_{\mathrm{GW}}\left(\nu, \tau_{0}\right) d \ln \nu \leq 8.7 \times 10^{-6} .
$$

While the bound itself is numerically similar to the one of Eq. (4.13), the lower extremum of integration is smaller since $\nu_{\mathrm{dec}} \ll \nu_{b b n}$ [see Eqs. (4.8) and (4.9)]. The bound (4.14) (discussed in Ref. [62] with slightly different notations) has been also taken into account in the present analysis. However, since we are dealing here with growing spectral energy distributions, Eq. (4.14) is less constraining: for the same (increasing) slope the lower extremum of integration of Eq. (4.14) gives a smaller contribution than the one of Eq. (4.13).

\section{Spectral energy distribution}

The analytic estimates of the spectral energy density of Sec. III lead to approximate expressions of the spectral energy distribution; however, for a more quantitative 
assessment the cosmic graviton spectrum should be expressed in terms of $T_{\text {eq }}\left(\nu, \nu_{\mathrm{eq}}\right), T_{*}\left(\nu, \nu_{*}\right)$, and $T_{s}\left(\nu, \nu_{s}\right)$ denoting, respectively, the transfer functions of the energy density at low, intermediate, and high frequencies:

$$
\begin{aligned}
T_{\mathrm{eq}}\left(\nu, \nu_{\mathrm{eq}}\right) & =\sqrt{1+c_{\mathrm{eq}}\left(\frac{\nu_{\mathrm{eq}}}{\nu}\right)+b_{\mathrm{eq}}\left(\frac{\nu_{\mathrm{eq}}}{\nu}\right)^{2}} \\
c_{\mathrm{eq}} & =0.5238, \quad b_{\mathrm{eq}}=0.3537 \\
T_{*}\left(\nu, \nu_{*}\right) & =\left[1+c_{*}\left(\frac{\nu}{\nu_{*}}\right)^{2 \epsilon+n_{T}}+b_{*}\left(\frac{\nu}{\nu_{*}}\right)^{4 \epsilon+2 n_{T}}\right]^{-1 / 2} \\
c_{*} & =b_{*}=\mathcal{O}(1), \\
T_{s}\left(\nu, \nu_{s}\right) & =\sqrt{1+c_{s}\left(\frac{\nu}{\nu_{s}}\right)^{p(w) / 2}+b_{s}\left(\frac{\nu}{\nu_{s}}\right)^{p(w)}} \\
p(w) & =2-\frac{4}{3 w+1},
\end{aligned}
$$

where the subscripts refer to the typical frequencies involved in each transition, i.e., $\nu_{\mathrm{eq}}, \nu_{*}$, and $\nu_{s}$. To transfer the spectral energy density inside the Hubble radius, the procedure is to integrate numerically the equations of the tensor modes; the derivation of $T_{\text {eq }}\left(\nu, \nu_{\text {eq }}\right)$ and $T_{s}\left(\nu, \nu_{s}\right)$, in a different physical situation, has been discussed in detail in $[50,63,64]$. In the literature it is also customary to introduce the transfer function of the power spectrum $[65,66]$ and the two transfer functions have slightly different numerical features that have been discussed in the past (see, e.g., Ref. [50] for a comparison). With these specifications, we have

$$
\begin{aligned}
& h_{0}^{2} \Omega_{g w}\left(\nu, \tau_{0}\right)=\mathcal{N}_{\rho} r_{T}\left(\nu_{p}\right) \mathcal{T}^{2}\left(\nu, \nu_{\mathrm{eq}}, \nu_{*}, \nu_{s}\right)\left(\frac{\nu}{\nu_{\mathrm{p}}}\right)^{n_{\mathrm{T}}} e^{-2 \beta \nu / \nu_{\max }}, \\
& \mathcal{T}\left(\nu, \nu_{\mathrm{eq}}, \nu_{*}, \nu_{s}\right)=T_{\mathrm{eq}}\left(\nu, \nu_{\mathrm{eq}}\right) T_{*}\left(\nu, \nu_{*}\right) T_{s}\left(\nu, \nu_{s}\right) \\
& \mathcal{N}_{\rho}=4.165 \times 10^{-15}\left(\frac{h_{0}^{2} \Omega_{\mathrm{R} 0}}{4.15 \times 10^{-5}}\right)\left(\frac{\mathcal{A}_{\mathcal{R}}}{2.41 \times 10^{-9}}\right)
\end{aligned}
$$

where $n_{T}=[\alpha(3-2 \gamma)-2 \epsilon] /(1+\alpha-\epsilon) \quad$ [see also Eq. (3.35)] and $r_{T}\left(\nu_{p}\right)$ is the tensor to scalar ratio of Eq. (3.14) evaluated at the pivot frequency $\nu_{p}$. In the conventional case $r_{T}\left(\nu_{p}\right)$ is related to the slow-roll parameter $\epsilon$ and to the tensor spectral index $n_{T}$ via the so-called consistency relations (see also, in this respect, Ref. [67] where a similar model for the violation of the consistency relations has been discussed). In the present situation $r_{T}(\nu)$ and $n_{T}$ do not obey the consistency relations and depend on the rate of variation of the refractive index $\alpha$ and on the critical number of $e$-folds $N_{*}$. If the refractive index is not dynamical (i.e., $\alpha \rightarrow 0$ and $\gamma \rightarrow 0$ ) we have, as expected, that
$n_{T} \rightarrow-2 \epsilon$. In Eq. (4.18) $\beta$ is a parameter $\mathcal{O}(1)$ which depends upon the width of the transition between the inflationary phase and the subsequent radiation-dominated phase; for different widths of the post-inflationary transition we can estimate $0.5 \leq \beta \leq 6.3$ [63,64]. The numerical coefficients appearing in Eqs. (4.15)-(4.17) are determined from each specific transition: while $c_{\text {eq }}$ and $b_{\text {eq }}$ can be accurately assessed, $c_{*}$ and $b_{*}$ depend on the parametrization of the refractive index and, similarly, $c_{s}$ and $b_{s}$ change depending on the values of $w$. In the case $w \rightarrow 1$ there are even logarithmic corrections which have been specifically scrutinized in the past ${ }^{15}$ (see, e.g., [32]).

\section{E. The constrained parameter space}

We shall be predominantly interested in the possibility of a relatively strong signal in the audio and in the $\mathrm{mHz}$ bands. We recall that the audio band ranges between a few $\mathrm{Hz}$ and $10 \mathrm{kHz}$ where the terrestrial wide-band interferometers operate. The $\mathrm{mHz}$ band ranges instead between a fraction of the $\mathrm{mHz}$ and the $\mathrm{Hz}$; in this range space-borne detectors might one day operate, hopefully within the following score year. The MHz band extends between $100 \mathrm{kHz}$ and a few $\mathrm{GHz}$; this band is immaterial for potential signals coming from conventional inflationary models but could play a relevant role since most of the signals discussed in the present paper are concentrated exactly in this region.

The results discussed hereunder refer predominantly ${ }^{16}$ to the case where the action for the relic gravitons is parametrized as in Eqs. (2.1) and (2.3). In the different figures reported below, the constrained parameter space will also be illustrated, in some cases, when $\gamma=1$. To account for the possibility of a detection in the audio band we shall then impose on the parameter space a further constraint on the chirp amplitude:

$$
h_{c}\left(\nu_{\text {audio }}, \tau_{0}\right)>10^{-25}, \quad \nu_{\text {audio }}=0.1 \mathrm{kHz},
$$

where $\nu_{\text {audio }}$ roughly corresponds, in practice, to the expected maximum of the sensitivity for the (advanced) Ligo/Virgo interferometers. In the $\mathrm{mHz}$ band we shall instead require

$$
h_{c}\left(\nu_{\mathrm{mHz}}, \tau_{0}\right)>2 \times 10^{-23}, \quad \nu_{\mathrm{mHz}}=\mathrm{mHz} .
$$

\footnotetext{
${ }^{15}$ According to Eq. (4.15), $T_{\text {eq }}(\nu) \rightarrow 1$ for $\nu \gg \nu_{\text {eq }}$ but in the realistic situations further suppressions are expected. The neutrino free streaming produces an effective anisotropic stress leading ultimately to an integro-differential equation (see, for instance, [12,13]). This aspect will be discussed later when assessing the other minor sources of damping.

${ }^{16}$ As discussed in Appendix C, the action of relic gravitons can be parametrized in different ways depending upon the value of a parameter $\gamma$ that appears in the generalized action of Eq. (C1). It is useful, for quantitative reasons, to compare the cases $\gamma=0$ [corresponding exactly to Eqs. (2.3) and (2.1)] and $\gamma=1$.
} 

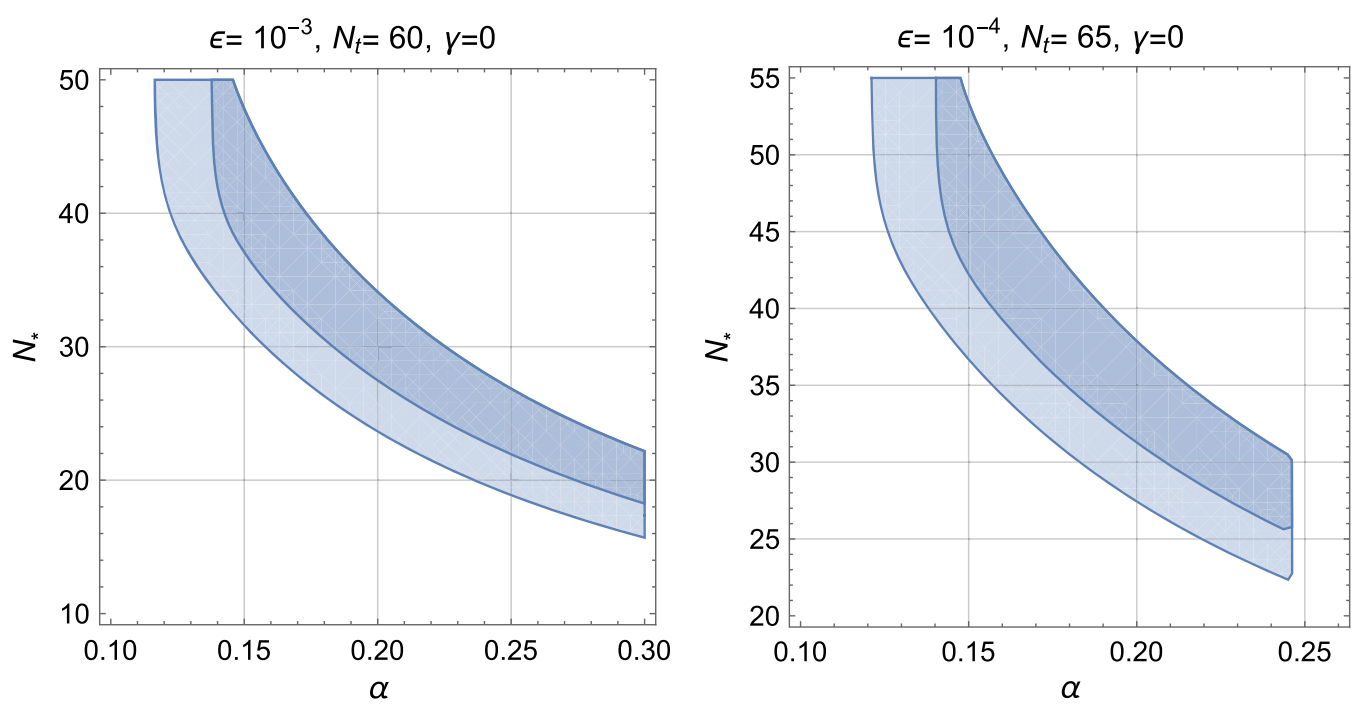

FIG. 4. The outer and the inner shaded areas illustrate the regions of the parameter space where all the constraints are satisfied in conjunction either with the requirements of Eqs. (4.23) and (4.24) (outer regions) or with the stronger demands of Eqs. (4.25) and (4.26) (inner regions). Both plots refer to the case where the graviton action is parametrized as in Eqs. (2.3) and (2.1) [i.e., $\gamma=0$ in the language of the generalized parametrization of Eq. (C1).

Equations (4.21) and (4.22) imply that we should select regions of the parameter space where the spectral energy distribution exceeds, respectively, $10^{-11}$ and $10^{-16}$; more specifically we are led to demand

$$
\begin{gathered}
h_{0}^{2} \Omega_{g w}\left(\nu_{\text {audio }}, \tau_{0}\right)>6.2 \times 10^{-11}, \quad \nu_{\text {audio }}=0.1 \mathrm{kHz}, \\
h_{0}^{2} \Omega_{g w}\left(\nu_{\mathrm{mHz}}, \tau_{0}\right)>2.5 \times 10^{-16}, \quad \nu_{\mathrm{mHz}}=\mathrm{mHz} .
\end{gathered}
$$

Since these requirements might not be achieved with rushing speed, we shall also consider a couple of less pretentious conditions, namely,

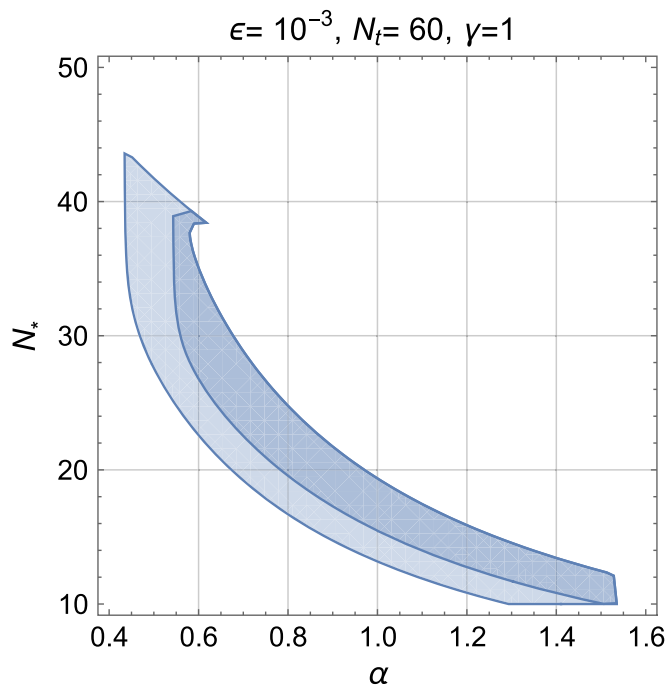

$$
\begin{array}{r}
h_{0}^{2} \Omega_{g w}\left(\nu_{\text {audio }}, \tau_{0}\right)>10^{-9}, \quad \nu_{\text {audio }}=0.1 \mathrm{kHz}, \\
h_{0}^{2} \Omega_{g w}\left(\nu_{\mathrm{mHz}}, \tau_{0}\right)>10^{-12}, \quad \nu_{\mathrm{mHz}}=\mathrm{mHz} .
\end{array}
$$

While from the viewpoint of the experiments, Eqs. (4.25) and (4.26) are weaker than Eqs. (4.23) and (4.24), from the viewpoint of the signal itself the opposite is true: if we enforce Eqs. (4.23) and (4.24), the allowed region of the parameter space will be larger than in the case of Eqs. (4.25) and (4.26). See, in this respect, Figs. 4 and 5 where we illustrate the constrained parameter space for different

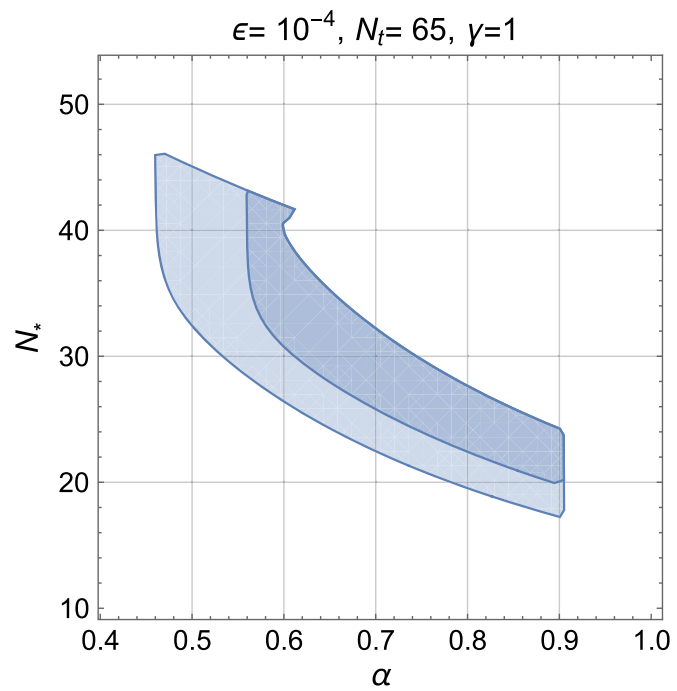

FIG. 5. For quantitative comparison it is interesting to illustrate the same analysis of Fig. 4 in the case $\gamma=1$. This case corresponds to a different parametrization of the graviton action [see Appendix $\mathrm{C}$ and, in particular, Eq. $(\mathrm{C} 1)$ ] that has been invoked by some authors to propose a purportedly different mechanism for the evolution of the refractive index. On the contrary, as argued in the Appendix, these are just two conformally equivalent representations of the original action of Eqs. (2.3) and (2.1). 

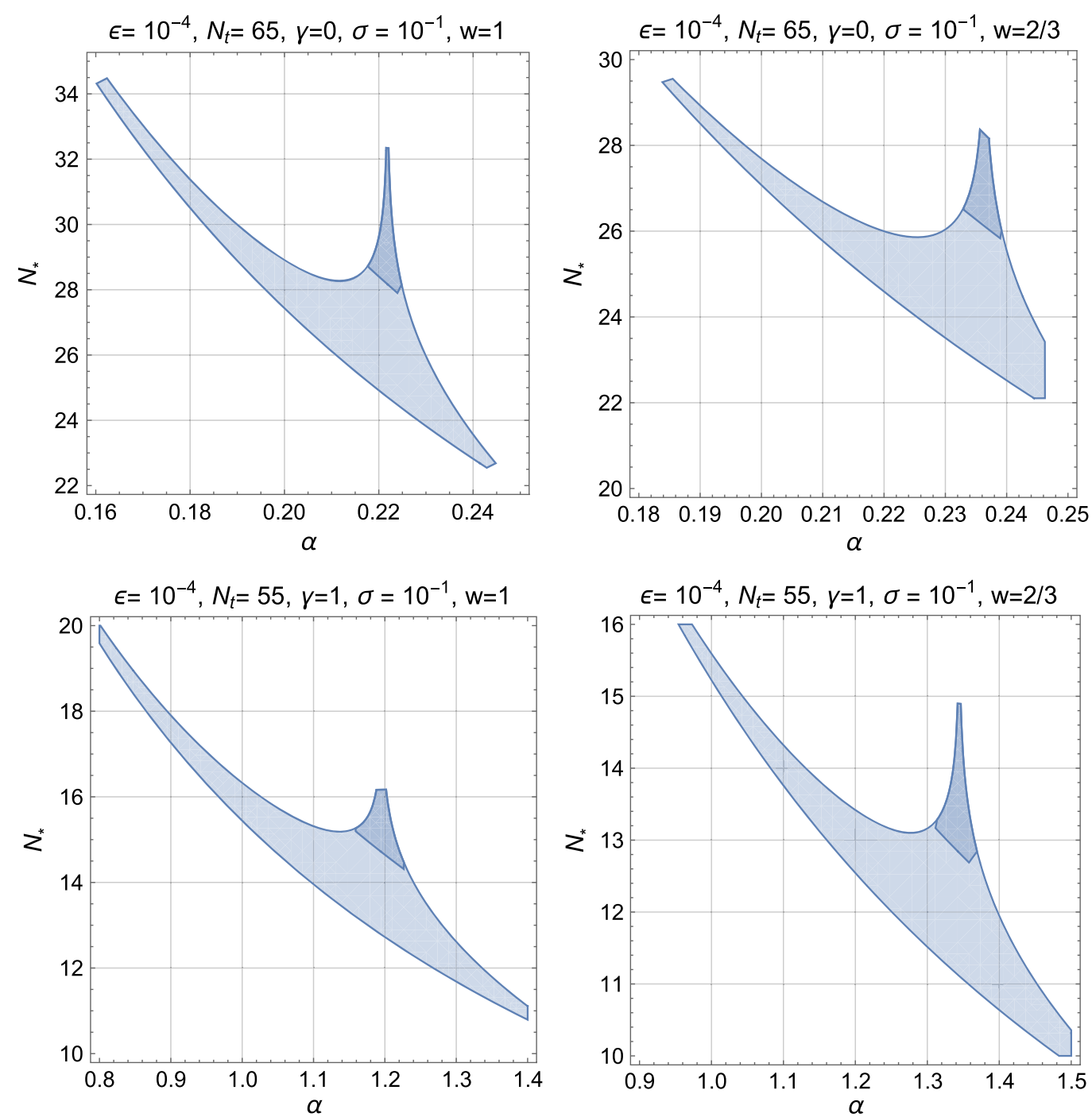

FIG. 6. We illustrate the constrained parameter space in the case of a thermal history characterized by a stiff post-inflationary phase. As in the case of Figs. 4 and 5, the outer and the inner regions refer, respectively, to the requirements of Eqs. (4.23) and (4.24) and Eqs. (4.25) and (4.26).

choices of the parameters and in the case of a conventional thermal history. The shaded areas in both plots describe the regions where all the phenomenological constraints are concurrently satisfied while the chirp amplitudes are sufficiently large enough to be detected. More specifically, in Figs. 4 and 5 the outer regions are obtained by enforcing the requirements of Eqs. (4.25) and (4.26). Conversely the inner regions come from the more demanding conditions spelled out in Eqs. (4.23) and (4.24). The reduction of the areas between the outer and the inner regions illustrate the reduction of the parameter space induced by the difference between the requirements of Eqs. (4.23) and (4.24) and Eqs. (4.25) and (4.26).

In the case of a different post-inflationary history, the constrained parameter space gets modified and the relevant exclusion plots are illustrated in Fig. 6 for a fiducial choice of the parameters. In the two plots at the right, the barotropic index corresponds to $2 / 3$ while in the two plots at the left, the barotropic index is maximal (i.e., $w=1$ ). By looking at the inner and at the outer exclusion regions we conclude that a reduction in the sensitivities of the hypothetical detectors drastically reduces the areas of the parameter space. Indeed, as in Figs. 4 and 5, the inner and the outer plots correspond, respectively, to the requirements of Eqs. (4.25) and (4.26) and to the requirements of Eqs. (4.23) and (4.24). The reason for this reduction is a direct consequence of the violet spectral slope in the highest frequency domain. Still, for a given value of $\sigma$, the constrained parameter space suggests a potentially interesting signal.

The explicit profiles of different models will now be illustrated. While the same analysis can be easily rephrased either in terms of the power spectrum $\mathcal{P}_{T}\left(\nu, \tau_{0}\right)$ or in terms of the spectral amplitude $S_{h}\left(\nu, \tau_{0}\right)$ we shall be mainly 

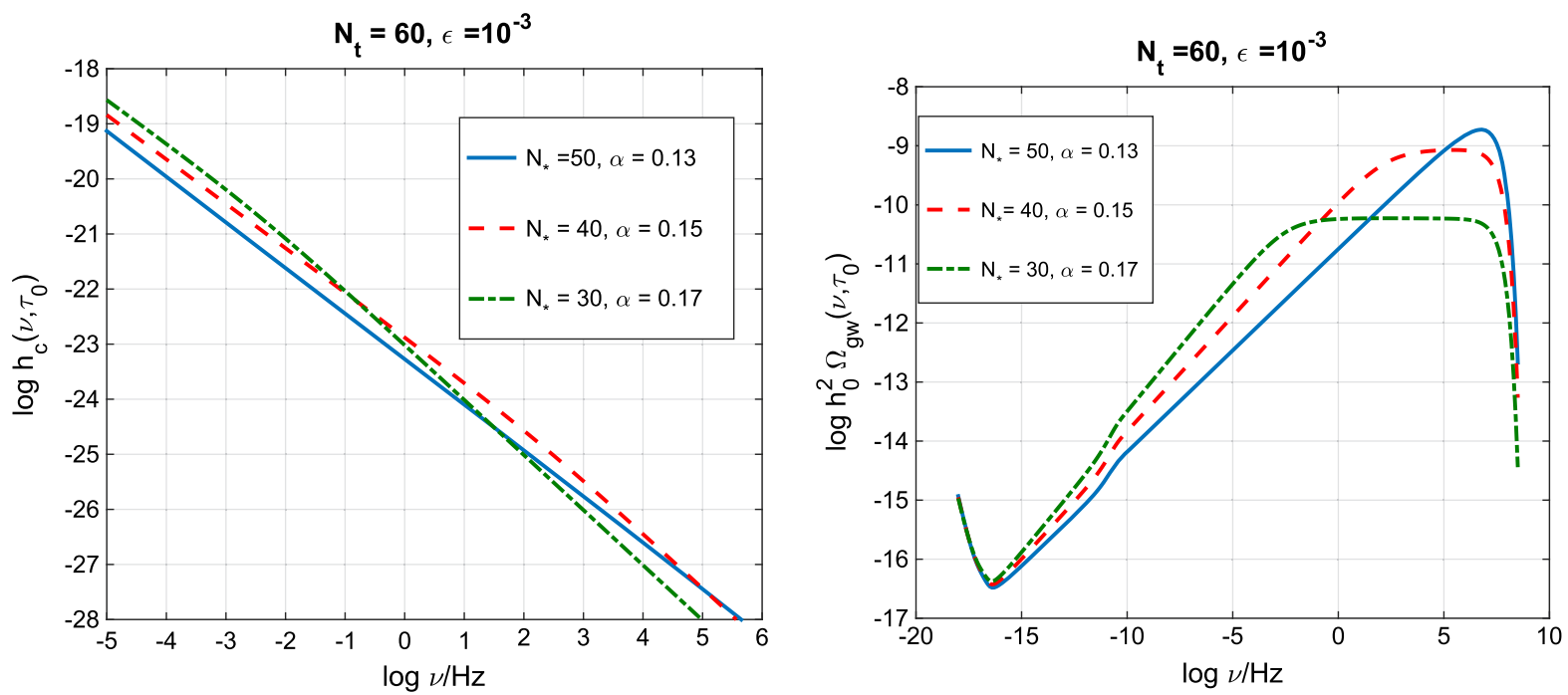

FIG. 7. The chirp amplitude and the spectral energy distribution produced by a dynamical refractive index are illustrated in the case of a standard post-inflationary thermal history. Note that in this and in the following two figures we took $\gamma=0$.
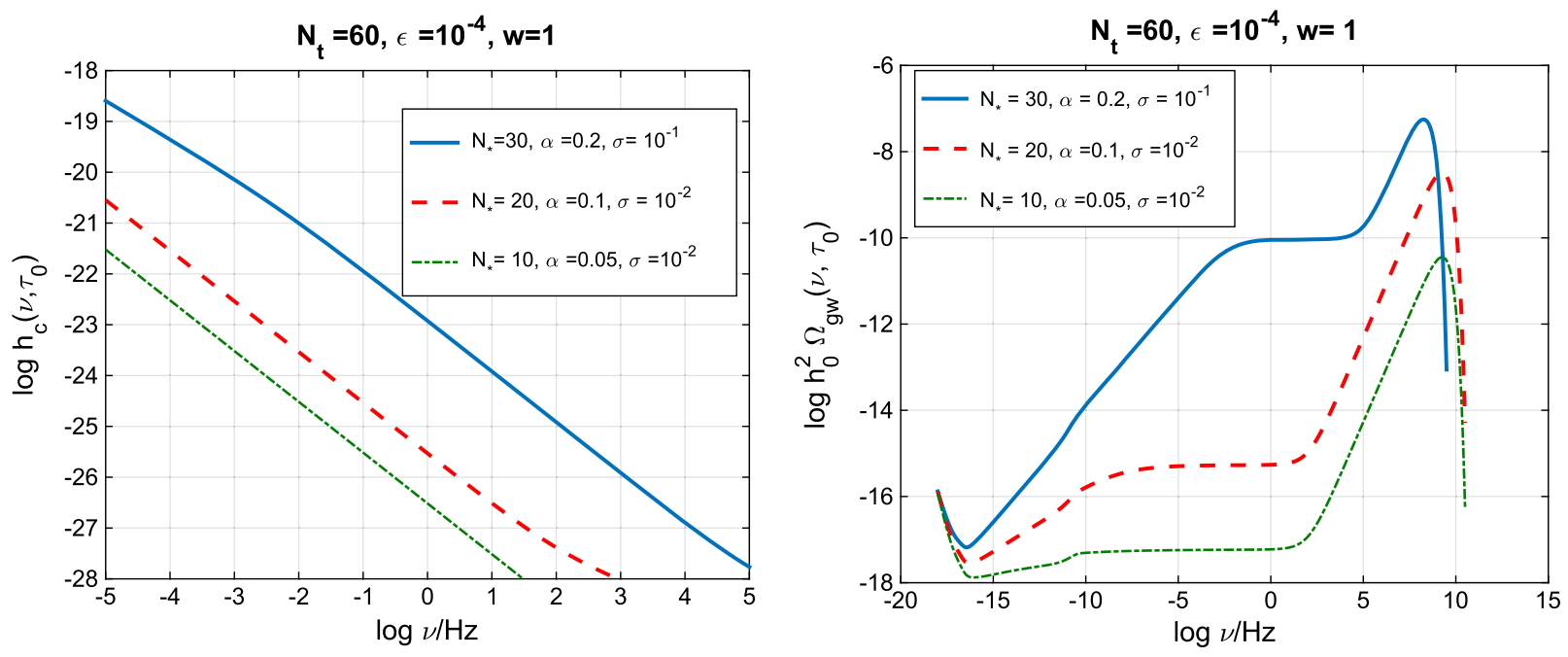

FIG. 8. The relic graviton background is illustrated in the case of a dynamical refractive index and assuming a post-inflationary thermal history that includes a stiff phase with $w=1$.

interested in the chirp amplitude and in the spectral energy distribution. This kind of approach is also useful for the explicit derivation of a template family.

In Fig. 7 we illustrate the chirp amplitude and the spectral energy distribution in the case of a standard post-inflationary history. The scales of the two plots on the horizontal axis are different. ${ }^{17}$ The explicit differences among the various models are less pronounced if we look at the chirp amplitude which is proportional to the square root of the spectral energy distribution and it is further

\footnotetext{
${ }^{17}$ The chirp amplitude is illustrated in a frequency range encompassing the $\mathrm{mHz}$ and the audio bands. Conversely, the spectral energy distribution covers all the frequencies from the $\mathrm{aHz}$ up to the $\mathrm{MHz}$ band.
}

suppressed by one power of the frequency. As $N_{*}$ increases, the plateau of Fig. 7 becomes less evident. The results of Fig. 7 can be usefully compared with the ones illustrated in Fig. 8 where the post-inflationary evolution is characterized by a stiff epoch. While in Fig. 8 we considered the case $w=1$, in Fig. 9 we took instead $w=2 / 3$. Recalling Eqs. (3.37) and (3.38), and (4.12), the value of the barotropic index controls not only the slope of the cosmic graviton spectrum in the vicinity high-frequency spike but also the frequency range. Larger values of $w$ correspond to more violet slopes in the $\mathrm{MHz}$ band while the values of $\sigma$ are inversely proportional to the frequency of the spike so that as $\sigma$ gets smaller than 1 the position of the spike exceeds the GHz. Both $\sigma$ and $w$ determine the length of the stiff phase. 

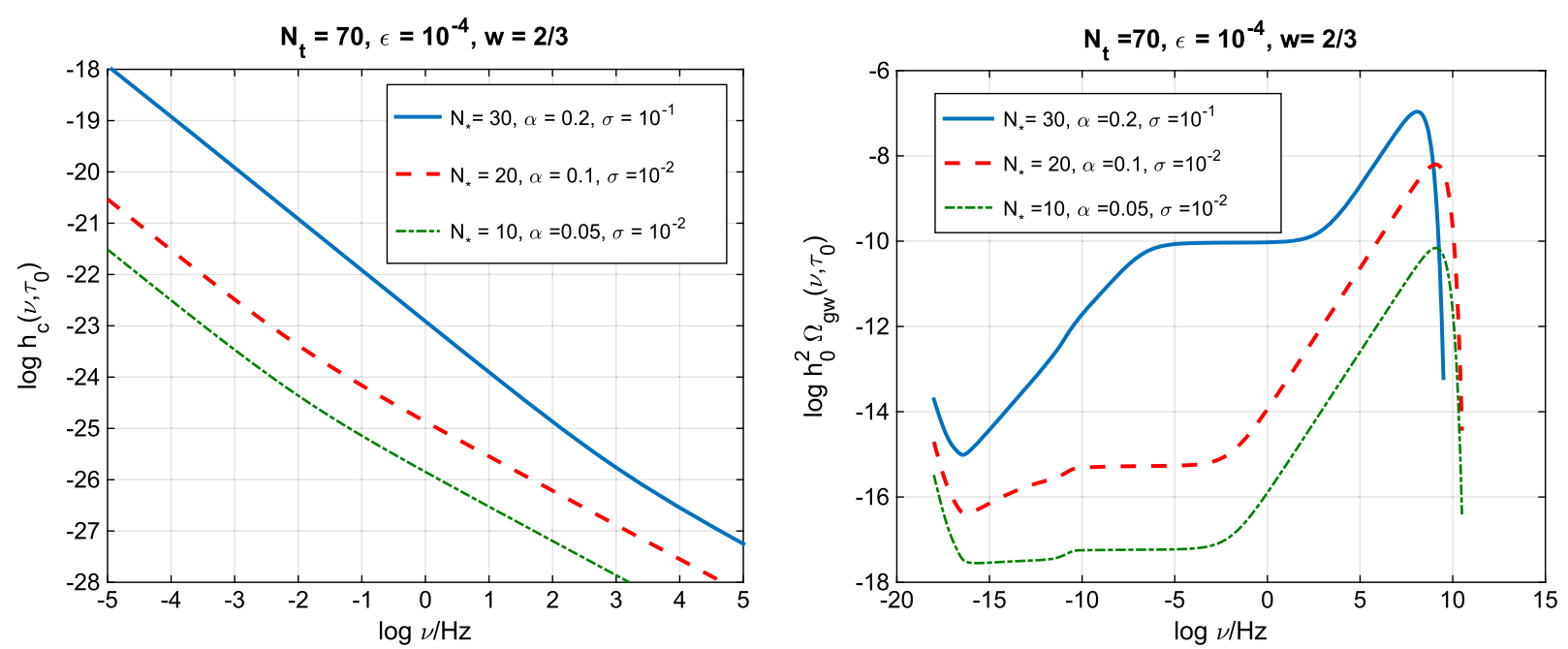

FIG. 9. The same analysis of Fig. 8 is repeated in the case $w=2 / 3$ for slightly different values of the parameters to illustrate potential degeneracies in the parameter space.

In the right plots of Figs. 7-9 we can appreciate a minor suppression for frequencies of the order of $0.01 \mathrm{nHz}$. This suppression is less visible in the chirp amplitude but it is evident from the spectral energy distribution. For $\nu<\nu_{b b n}$, the slight break in the spectrum is due to the neutrino free streaming. The neutrinos free stream, after their decoupling, and the effective energy-momentum tensor acquires, to first-order in the amplitude of the plasma fluctuations, an anisotropic stress. The overall effect of collisionless particles is a reduction of the spectral energy density of the relic gravitons. Assuming that the only collisionless species in the thermal history of the Universe are the neutrinos, the amount of suppression can be parametrized by the function

$$
\begin{aligned}
\mathcal{F}\left(R_{\nu}\right) & =1-0.539 R_{\nu}+0.134 R_{\nu}^{2}, \\
R_{\nu} & =\frac{r}{r+1}, \quad r=0.681\left(\frac{N_{\nu}}{3}\right),
\end{aligned}
$$

where, as usual, $R_{\nu}$ is the fraction of neutrinos in the radiation plasma; clearly in the concordance model $R_{\gamma}+R_{\nu}=1$. In the case $R_{\nu}=0$ (i.e., in the absence of collisionless particles) there is no suppression. If, on the contrary, $R_{\nu} \neq 0$ the suppression can even reach 1 order of magnitude. In the case $N_{\nu}=3, R_{\nu}=0.405$ and the suppression of the spectral energy density is proportional to $\mathcal{F}^{2}(0.405)=0.645$. This suppression due to neutrino free streaming is thus effective for frequencies larger than $\nu_{\mathrm{eq}}$ and smaller than $\nu_{b b n}$.

Besides neutrino free streaming, there are other two minor effects taken into account in Figs. 7-9: the damping effect associated with the (present) dominance of the dark energy and the suppression due to the variation of the effective number of relativistic species. In the concordance scenario, the redshift of $\Lambda$ dominance [i.e., $\left(\Omega_{\Lambda} / \Omega_{M 0}\right)^{1 / 3}$ ] determines the numerical value of $\nu_{\Lambda}$ defined in Eq. (4.7).
The adiabatic damping of the mode function due to the dominance of the dark energy implies a damping of the order of $\left(\Omega_{M 0} / \Omega_{\Lambda}\right)^{2}$ in the spectral energy distribution. This suppression competes with a potential increase of the spectral energy distribution for $\nu<\nu_{\lambda}$ and going as $\left(\nu / \nu_{\Lambda}\right)^{-2}$ $[14,68]$. Finally, for temperatures much larger than the top quark mass, all the known species of the minimal standard model of particle interactions are in local thermal equilibrium, then $g_{\rho}=g_{\mathrm{s}}=106.75$. Below $T \simeq 175 \mathrm{GeV}$, the various species start decoupling, the notion of thermal equilibrium is replaced by the notion of kinetic equilibrium and the time evolution of the number of relativistic d.o.f. effectively changes the evolution of the Hubble rate. In principle, if a given mode $k$ reenters the Hubble radius at a temperature $T_{k}$ the spectral energy density of the relic gravitons is (kinematically) suppressed by a factor which can be written as $[63,64]\left(g_{\rho}\left(T_{k}\right) / g_{\rho 0}\right)\left(g_{\mathrm{s}}\left(T_{k}\right) / g_{\mathrm{s} 0}\right)^{-4 / 3}$ where, at the present time, $g_{\rho 0}=3.36$ and $g_{\mathrm{s} 0}=3.90$. So, in the case of the minimal standard model the suppression on $\Omega_{g w}\left(\nu, \tau_{0}\right)$ will be of the order of 0.38. In popular supersymmetric extensions of the minimal standard models, $g_{\rho}$ and $g_{s}$ can be as high as, approximately, 230. This will bring down the figure given above to 0.29 .

\section{CONCLUDING REMARKS}

The evolution of the refractive index during the early stages of a conventional inflationary phase leads to a spectral energy distribution naturally tilted towards frequencies and different post-inflationary thermal histories typically add a further branch to the cosmic graviton spectrum. Overall the spectrum may then exhibit up to four different branches extending between the $\mathrm{aHz}$ region and the $\mathrm{GHz}$ band. Assuming, in a minimalistic perspective, that the evolution of the refractive index terminates before the end of inflation, 
the spectral energy distribution involves a quasiflat plateau at high frequencies that is supplemented by a spike between the $\mathrm{MHz}$ and the GHz. Depending on the thermal history, the slopes of the spectral energy distribution are red, blue, and even violet. After imposing the usual phenomenological constraints, there are still wide portions of the parameter space where the resulting signal could be detectable, at least, in principle, either by terrestrial interferometers (in their advanced and enhanced configuration) or by space-borne detectors. In spite of less mundane possibilities leading to growing spectral energy distributions of relic gravitons, the present findings demonstrate that blue and violet spectra are compatible with conventional inflationary scenarios in the presence of a dynamical refractive index.

\section{ACKNOWLEDGMENTS}

I wish to thank M. Doser, F. Fidecaro, M. Pepe-Altarelli, and $\mathrm{G}$. Unal for interesting exchanges on the topics discussed in this paper. I also thank T. Basaglia, A. Gentil-Beccot, and S. Rohr of the CERN Scientific Information Service for their kind assistance.

\section{APPENDIX A: DIFFERENT PARAMETERIZATION OF THE GRAVITON ACTION}

Gravitational waves might acquire a refractive index when they evolve in curved space-times [30,31] and the impact of this idea on a quasi-de Sitter stage of expansion has been explored in [32] where the presence of a (time dependent) refractive index has been introduced for the first time. For standard dispersion relations the propagating speed of the tensor modes of the geometry in natural units coincides with the inverse of the refractive index [i.e., $\left.c_{g w}(\tau)=1 / n(\tau)\right]$ and the basic action can be written, in a covariant language, as

$$
\begin{aligned}
S^{(E)}= & \frac{1}{8 \ell_{P}^{2}} \int d^{4} x \sqrt{-\bar{g}_{(E)}}\left[\bar{g}_{(E)}^{\mu \nu} \partial_{\mu} h_{i j}^{(E)} \partial_{\nu} h_{i j}^{(E)}\right. \\
& \left.+\left(\frac{1}{n^{2}}-1\right) \bar{P}_{(E)}^{\mu \nu} \partial_{\mu} h_{i j}^{(E)} \partial_{\nu} h_{i j}^{(E)}\right],
\end{aligned}
$$

where $\ell_{P}=\sqrt{8 \pi G}=1 / \bar{M}_{P}$ and $\bar{P}_{(E)}^{\mu \nu}$ is the spatial projector tensor orthogonal to $\bar{u}_{\mu}^{(E)}$ :

$$
\bar{P}_{(E)}^{\mu \nu}=\bar{g}_{(E)}^{\mu \nu}-\bar{u}_{(E)}^{\mu} \bar{u}_{(E)}^{\nu}, \quad \bar{g}_{(E)}^{\mu \nu} \bar{u}_{\mu}^{(E)} \bar{u}_{\nu}^{(E)}=1 .
$$

In the case $n \rightarrow 1$ the action of Eq. (A1) reproduces the original Ford and Paker result [3]; in comoving coordinates ${ }^{18}$ and in a conformally flat metric Eq. (A1) assumes the following form:

\footnotetext{
${ }^{18}$ In the case of a conformally flat metric $\bar{g}_{\mu \nu}^{(E)}=a_{E}^{2} \eta_{\mu \nu}$ (where $\eta_{\mu \nu}$ is the Minkowski metric) we have $\bar{u}_{(E)}^{0}=1 / a$.
}

$$
S^{(E)}=\frac{1}{8 \ell_{P}^{2}} \int d^{3} x \int d \tau a_{E}^{2}\left[\partial_{\tau} h_{i j}^{(E)} \partial_{\tau} h_{i j}^{(E)}-\frac{1}{n^{2}} \partial_{k} h_{i j}^{(E)} \partial^{k} h_{i j}^{(E)}\right] .
$$

Equation (A3) coincides exactly with Eq. (2.1) but it is written here in a way that makes clearly apparent the frame where it is written. The inverse of the refractive index multiplies each spatial derivative of the tensor amplitude [see Eq. (A3)]. This is the parametrization employed in Refs. [30-32] and it is physically motivated. It is, however, possible to adopt a somehow contrived viewpoint and to describe the dynamics of the refractive index with an apparently different action, namely,

$$
S=\frac{1}{8 \ell_{P}^{2}} \int d^{3} x \int d \tau a^{2} n^{2}\left[\partial_{\tau} h_{i j} \partial_{\tau} h_{i j}-\frac{1}{n^{2}} \partial_{k} h_{i j} \partial^{k} h_{i j}\right]
$$

To get from Eq. (A3) to Eq. (A4) we need a specific transformation that leaves unaltered the conformal time coordinate and the tensor amplitude while the scale factor is simply rescaled through the refractive index itself:

$$
a_{E} \rightarrow a=\frac{a_{E}}{n}, \quad h_{i j}^{(E)} \rightarrow h_{i j}=h_{i j}^{(E)} .
$$

This transformation exists and it is nothing but a conformal rescaling. We shall now specifically discuss how to pass from Eq. (A3) to (A4). The bottom line of this discussion is that Eq. (A4) has the same physical content of Eqs. (2.1) and (A3) but it is written in a conformally related frame. For instance, the authors of Ref. [51] claim that their action is different from the one of Ref. [32] [which coincides with the canonical action of Eq. (2.1)] just because they write it in a conformally related frame. This way of proceeding, as we shall argue, is deliberately confusing and demonstrates that the results of [51] recycle the analysis of [32] by using a different frame.

\section{APPENDIX B: GAUGE-INVARIANCE AND FRAME INVARIANCE}

Indeed, Eq. (A5) transforms separately the background and the tensor inhomogeneities but it is not difficult to see that it comes directly from a conformal rescaling that leaves unaltered the tensor fluctuations of the geometry. To make this point more apparent, let us verify explicitly that the transformation (A5) is just a particular case of the following conformal rescaling of the four-dimensional metric:

$$
g_{\mu \nu}^{(E)}=\Omega^{2} G_{\mu \nu}, \quad \sqrt{-g^{(E)}}=\Omega^{4} \sqrt{-G},
$$


that implies the transformation (A5) on the background and on the related inhomogeneities. ${ }^{19}$

The second relation reported in Eq. (A5) is also a general consequence of the conformal rescaling (B1). While the proof of this statement is immediate in the case of the tensor modes, it is useful to present the complete argument since there are also symmetric implications in the case of the scalar modes. Neglecting, for simplicity, the vector modes of the geometry, the fluctuations of the metric in the Einstein frame are

$$
g_{\mu \nu}^{(E)}(\vec{x}, \tau)=\bar{g}_{\mu \nu}^{(E)}(\tau)+\delta_{t} g_{\mu \nu}^{(E)}(\vec{x}, \tau)+\delta_{s} g_{\mu \nu}^{(E)}(\vec{x}, \tau),
$$

where $\delta_{t} g_{\mu \nu}^{(E)}$ and $\delta_{s} g_{\mu \nu}^{(E)}$ denote, respectively, the tensor and the scalar fluctuations of the geometry in the Einstein frame. In a conformally flat background geometry of THE Friedmann-Robertson-Walker type, Eq. (B2) becomes

$$
\begin{gathered}
\delta_{\mathrm{t}} g_{i j}^{(E)}=-a_{E}^{2}(\tau) h_{i j}^{(E)}, \quad \partial_{i} h_{(E)}^{i j}=h_{(E) i}^{i}=0, \\
\delta_{s} g_{00}^{(E)}=2 a_{E}^{2} \phi_{E}, \quad \delta_{s} g_{i j}^{(E)}=2 a_{E}^{2}\left(\psi_{E} \delta_{i j}-\partial_{i} \partial_{j} C_{E}\right), \\
\delta_{s} g_{0 i}^{(E)}=-a_{E}^{2} \partial_{i} B_{E},
\end{gathered}
$$

where $h_{i j}^{(E)}$ is the (divergenceless and traceless) tensor amplitude appearing in Eq. (A1). By definition, the tensor amplitude $h_{i j}^{(E)}$ is invariant under infinitesimal diffeomorphisms while the scalar fluctuations are not.

Let us now consider exactly the same decomposition in the conformally related frame defined by Eq. (B1); as in the case of Eq. (B2), the $G_{\mu \nu}$ can be decomposed into a homogeneous part supplemented by its own tensor inhomogeneities:

$$
G_{\mu \nu}(\vec{x}, \tau)=\bar{G}_{\mu \nu}(\tau)+\delta_{t} G_{\mu \nu}(\vec{x}, \tau)+\delta_{s} G_{\mu \nu}(\vec{x}, \tau),
$$

where this time the explicit form of the tensor and scalar fluctuations of the four-dimensional metric will be given by

$$
\begin{aligned}
& \delta_{t} G_{i j}=-a^{2} h_{i j}, \quad \partial_{i} h_{j}^{i}=h_{i}^{i}=0, \\
& \delta_{s} G_{00}=2 a^{2} \phi, \quad \delta_{s} G_{i j}=2 a^{2}\left(\psi \delta_{i j}-\partial_{i} \partial_{j} C\right), \\
& \delta_{s} G_{0 i}=-a^{2} \partial_{i} B .
\end{aligned}
$$

The tensor amplitude $h_{i j}$ defined in Eq. (B6) is gauge invariant while the scalar fluctuations of Eq. (B7) are not

\footnotetext{
${ }^{19}$ From Eq. (B1) the transformation for the background is immediate and it is given by $\bar{g}_{\mu \nu}^{(E)}=\Omega^{2} \bar{G}_{\mu \nu}$. In the case of a conformally flat metric of THE Friedmann-Robertson-Walker type, we have $\bar{g}_{\mu \nu}^{(E)}=a_{E}^{2}(\tau) \eta_{\mu \nu}$ and this means $\bar{G}_{\mu \nu}^{(E)}=a^{2}(\tau) \eta_{\mu \nu}$. Thus, since $a_{E}=\Omega a$, this transformation coincides exactly with the first relation of Eq. (A5) provided, as anticipated, $\Omega \equiv n$.
}

immediately gauge invariant. To work out the relation between the fluctuations in the two frames, we can therefore start with the tensor modes; from Eq. (B1), recalling the explicit forms of the tensor fluctuations in the two frames [i.e., Eqs. (B3) and (B6)] we can write

$$
\delta_{\mathrm{t}} g_{\mu \nu}^{(E)}=\Omega^{2} \delta_{t} G_{\mu \nu}, \quad \bar{g}_{\mu \nu}^{(E)}=\Omega^{2}(\tau) \bar{G}_{\mu \nu} .
$$

Inserting Eqs. (B3) and (B6) into Eq. (B8) we have, as anticipated, that

$$
h_{i j}^{(E)}=h_{i j}, \quad a_{E}(\tau)=\Omega(\tau) a(\tau),
$$

which coincides with the transformation posited in Eq. (A5) if and only if $\Omega=n$. It is therefore legitimate to conclude that if the two backgrounds are conformally related, the gauge-invariant tensor amplitudes are also the same in the two frames. In other words the tensor amplitudes defined as in Eqs. (B3) and (B6) are both gauge invariant and frame invariant. In the conformally related frame the action of Eq. (A1) becomes

$$
\begin{aligned}
S^{(E)} \rightarrow S= & \frac{1}{8 \ell_{P}^{2}} \int d^{4} x\left\{\sqrt { - \overline { G } } \left[\bar{G}^{\mu \nu} \Omega^{2} \partial_{\mu} h_{i j} \partial_{\nu} h_{i j}\right.\right. \\
& \left.\left.+\left(\frac{1}{n^{2}}-1\right) \Omega^{2} \bar{P}^{\mu \nu} \partial_{\mu} h_{i j} \partial_{\nu} h_{i j}\right]\right\},
\end{aligned}
$$

where the projectors and the four-velocities have been conformally rescaled as

$$
\begin{aligned}
\bar{P}_{(E)}^{\mu \nu} & =\frac{1}{\Omega^{2}} \bar{P}^{\mu \nu}, \quad \bar{P}^{\mu \nu}=\bar{G}^{\mu \nu}-\bar{U}^{\mu} \bar{U}^{\nu}, \\
\bar{G}_{\mu \nu} \bar{U}^{\mu} \bar{U}^{\nu} & =1, \quad \bar{U}^{\mu}=\frac{\bar{u}_{(E)}^{\mu}}{\Omega} .
\end{aligned}
$$

If we now posit that the conformal factor with the refractive index itself coincides [i.e., $\Omega(\tau)=n(\tau)$ ], the action of Eq. (B11) becomes exactly

$$
S=\frac{1}{8 \ell_{P}^{2}} \int d^{3} x \int d \tau a^{2}\left[n^{2}(\tau) \partial_{\tau} h_{i j} \partial_{\tau} h_{i j}-\partial_{k} h_{i j} \partial_{k} h_{i j}\right],
$$

which coincides with the action anticipated in Eq. (A4). All in all, the action of Eqs. (A3) and (A4) are one and the same action since they are simply related by a conformal rescaling.

It is interesting to mention, as we close the section, the analog results for the scalar modes of the geometry which are, however, less central to the discussion of the present investigation. Indeed, from Eq. (B1) we will have that

$$
\delta_{s} g_{\mu \nu}^{(E)}=\delta_{s} n \bar{G}_{\mu \nu}^{(s)}+n \delta_{s} G_{\mu \nu}
$$


Recalling then the explicit results of Eqs. (B4) and (B7), Eq. (B13) implies a specific relation between the perturbed components in the two frames, i.e.,

$$
\begin{aligned}
& \phi=\phi_{E}-\frac{1}{2}\left(\frac{\delta_{s} n}{n}\right), \quad \psi=\psi_{E}+\frac{1}{2}\left(\frac{\delta_{s} n}{n}\right), \\
& C=C_{E}, \quad B=B_{E} .
\end{aligned}
$$

Equation (B14) implies that, unlike their tensor counterparts, the scalar inhomogeneities defined in Eqs. (B4) and (B7) are neither gauge invariant nor frame invariant. Note, however, that the curvature perturbations on comoving orthogonal hypersurfaces are both gauge invariant and frame invariant. Indeed, in the two frames they are simply given by

$$
\mathcal{R}_{E}=-\psi_{E}-\frac{\mathcal{H}_{E}}{n^{\prime}} \delta_{s} n, \quad \mathcal{R}=-\psi-\frac{\mathcal{H}}{n^{\prime}} \delta_{s} n .
$$

Equation (B15) does not imply that $\mathcal{R}_{E} \neq \mathcal{R}$, as it could be superficially concluded. On the contrary, the mismatch between $\psi_{E}$ and $\psi$ is exactly compensated by the mismatch between $\mathcal{H}_{E}$ and $\mathcal{H}$. In fact, from the relation between the background scale factors (i.e., $a_{E}=a n$ ) we have $2\left(\mathcal{H}_{E}-\mathcal{H}\right)=n^{\prime} / n$ so that Eq. (B15) implies $\mathcal{R}=\mathcal{R}_{E}$. We therefore have, as anticipated, that the tensor modes of the geometry discussed in the bulk of the paper and the curvature perturbations on comoving orthogonal hypersurfaces are both frame invariant and gauge invariant. ${ }^{20}$

\section{APPENDIX C: GAUGE INVARIANCE AND FRAME INVARIANCE}

After the appearance of Ref. [32] [where the canonical action of Eq. (2.1) has been suggested] two similar papers [51] pursued the same idea. The two approaches ultimately coincide since they are related by a conformal rescaling involving the refractive index. To get from the description of Ref. [32] to the one of Ref. [51] it is sufficient to make a conformal rescaling and to parametrize the propagating speed or the refractive index as a power of the scale factor. Following the suggestion of Ref. [32], the authors of Ref. [51] considered the evolution of the refractive index in an inflating background. This choice is, however, potentially confusing: since the two descriptions are related by a conformal rescaling, the two backgrounds should also be conformally related [70]. This would mean, in practice, that if the background inflates in the Einstein frame, it might not inflate in the conformally related frame. However, since the choice of the pivotal frame where the background inflates is not constrained, the choice of Ref. [51] is, in a sense, mathematically legitimate but physically superficial especially in light of the previous literature. We are therefore in the situation where the two

\footnotetext{
${ }^{20}$ Note that this property has relevant implications in the context of some specific class of bouncing models such as the ones proposed in [69].
}

conformally related actions are simply two complementary parametrizations of the same effect.

To account for the different (and sometimes unwise) choices of the present literature, we can define a generalized action for the tensor modes encompassing the various possibilities suggested so far. If we wish to analyze different conformally related actions in the same framework we can use a trick and write

$S=\frac{1}{8 \ell_{P}^{2}} \int d^{3} x \int d \tau a^{2} n^{2 \gamma}\left[\partial_{\tau} h_{i j} \partial_{\tau} h_{i j}-\frac{1}{n^{2}} \partial_{k} h_{i j} \partial_{k} h_{i j}\right]$.

When $\gamma=0$, Eq. (C1) coincides with the action of Eq. (A1); conversely if $\gamma=1$ the action (C1) coincides instead with Eq. (B12). By keeping the value of $\gamma$ generic the two parametrizations can be compared in light of the present and future detectability prospects. Clearly different values of $\gamma$ affect the definition of the $\eta$ time introduced in Eq. (2.2); more specifically we will still have that $n(\eta) d \eta=$ $d \tau$ but, this time, $b(\eta)=a n^{\gamma-1 / 2}$ which coincides with Eq. (2.2) when $\gamma=0$. As a consequence also, Eq. (2.31) depends on the choice of $\gamma$. For instance if $\gamma=1$, Eq. (2.31) becomes $b(x)=a \sqrt{n} \propto x \sqrt{n_{*} x^{\alpha} e^{-\xi x}+1}$.

The value of $\gamma$ also modifies the definition of $\delta$ appearing in Eq. (2.35) and the explicit form of Eq. (2.38). More specifically we will have that, for a generic $\gamma$,

$b=a n^{\gamma-1 / 2}=b_{*}\left(\frac{a}{a_{*}}\right)^{\delta}, \quad b_{*}=a_{*} n_{*}^{\gamma-1 / 2}$,

$\delta=\alpha(\gamma-1 / 2)+1$.

As a consequence of Eq. (C2) the value of $\mu$ appearing in Eq. (3.6) is modified as $\mu=[3+2 \gamma \alpha-\epsilon] /[2(1+\alpha-\epsilon)]$. The explicit expressions of the power spectrum $\mathcal{P}_{T}\left(k, \eta_{*}\right)$ and of $q_{T}\left(n_{i}, N_{t}, N_{*}, n_{T}\right)$ of Eqs. (3.9) and (3.13) are then modified as

$$
\begin{aligned}
& \mathcal{P}_{T}\left(k, \eta_{*}\right)=\left(\frac{H_{*}}{M_{P}}\right)^{2} \frac{2^{6-n_{T}}}{\pi^{2}} \Gamma^{2}\left(\frac{3-n_{T}}{2}\right) n_{*}^{3-n_{T}-2 \gamma} \\
& \times\left|1+\frac{\alpha}{1-\epsilon}\right|^{2-n_{T}}\left(\frac{k}{a_{*} H_{*}}\right)^{n_{T}}, \\
& q_{T}\left(n_{i}, N_{t}, N_{*}, n_{T}\right) \\
& =\frac{2^{6-n_{T}}}{\pi^{2}} \Gamma^{2}\left(\frac{3-n_{T}}{2}\right) n_{i}^{3-n_{T}-2 \gamma} e^{\alpha N_{*}\left(3-2 \gamma-n_{T}\right)-n_{T}\left(N_{*}-N_{t}\right)} .
\end{aligned}
$$

The values of the spectral indices of Eqs. (3.35) and (3.38) are also affected by the different values of $\gamma$. Their general expressions are given by

$n_{T}=\frac{(3-2 \gamma) \alpha-2 \epsilon}{(1+\alpha-\epsilon)}=\frac{\alpha(3-2 \gamma)}{1+\alpha}+\frac{[-2+\alpha(1-2 \gamma)] \epsilon}{(1+\alpha)^{2}}+\mathcal{O}\left(\epsilon^{2}\right)$, 
$s_{T}=2-\frac{4}{3 w+1}+n_{T} \simeq 2-\frac{4}{3 w+1}+\frac{\alpha(3-2 \gamma)}{1+\alpha}+\mathcal{O}(\epsilon)$,

(C6) reproducing the results of Eqs. (3.35) and (3.38) when $\gamma \rightarrow 0$. When $\gamma \neq 0$ (and, in particular, when $\gamma=1$ ) the spectral index determined in the $\gamma=0$ case is just rescaled by a $\gamma$-dependent prefactor that can be reabsorbed in a redefinition of the spectral index.
[1] L. P. Grishchuk, Zh. Eksp. Teor. Fiz. 67, 825 (1974) [Sov. Phys. JETP 40, 409 (1975)].

[2] L. P. Grishchuk, Ann. N.Y. Acad. Sci. 302, 439 (1977).

[3] L. H. Ford and L. Parker, Phys. Rev. D 16, 1601 (1977).

[4] B. L. Hu and L. Parker, Phys. Lett. 63A, 217 (1977).

[5] A. A. Starobinsky, Pis'ma Zh. Eksp. Teor. Fiz. 30, 719 (1979) [JETP Lett. 30, 682 (1979)].

[6] V. A. Rubakov, M. V. Sazhin, and A. V. Veryaskin, Phys. Lett. 115B, 189 (1982).

[7] R. Fabbri and M. D. Pollock, Phys. Lett. 125B, 445 (1983).

[8] L. F. Abbott and M. B. Wise, Nucl. Phys. 244, 541 (1984).

[9] B. Allen, Phys. Rev. D 37, 2078 (1988).

[10] V. Sahni, Phys. Rev. D 42, 453 (1990).

[11] L. P. Grishchuk and M. Solokhin, Phys. Rev. D 43, 2566 (1991).

[12] S. Weinberg, Phys. Rev. D 69, 023503 (2004).

[13] D. A. Dicus and W. W. Repko, Phys. Rev. D 72, 088302 (2005).

[14] W. Zhao and Y. Zhang, Phys. Rev. D 74, 043503 (2006).

[15] M. Giovannini, Phys. Lett. B 668, 44 (2008).

[16] P. A. R. Ade et al. (BICEP2 and Keck Array Collaborations), Phys. Rev. Lett. 116, 031302 (2016).

[17] M. Giovannini, Phys. Lett. B 759, 528 (2016).

[18] G. Hinshaw et al. (WMAP Collaboration), Astrophys. J. Suppl. Ser. 208, 19 (2013).

[19] C. L. Bennett et al. (WMAP Collaboration), Astrophys. J. Suppl. Ser. 208, 20 (2013).

[20] W. J. Percival et al. Mon. Not. R. Astron. Soc. 401, 2148 (2010).

[21] R. Keisler et al., Astrophys. J. 743, 28 (2011).

[22] J. L. Sievers et al. (Atacama Cosmology Telescope Collaboration), J. Cosmol. Astropart. Phys. 10 (2013) 060.

[23] P. A. R. Ade et al. (Planck Collaboration), Astron. Astrophys. 594, A13 (2016).

[24] Y. B. Zeldovich, Mon. Not. R. Astron. Soc. 160, 1 (1972).

[25] B. Spokoiny, Phys. Lett. B 315, 40 (1993).

[26] M. Giovannini, Phys. Rev. D 58, 083504 (1998); Classical Quantum Gravity 16, 2905 (1999); Phys. Rev. D 60, 123511 (1999).

[27] P. J. E. Peebles and A. Vilenkin, Phys. Rev. D 59, 063505 (1999); H. Tashiro, T. Chiba, and M. Sasaki, Classical Quantum Gravity 21, 1761 (2004); T. J. Battefeld and D. A. Easson, Phys. Rev. D 70, 103516 (2004).

[28] D. Babusci and M. Giovannini, Phys. Rev. D 60, 083511 (1999); Classical Quantum Gravity 17, 2621 (2000); Int. J. Mod. Phys. D 10, 477 (2001).
[29] S. Weinberg, Cosmology (Oxford University Press, Oxford, 2008).

[30] P. Szekeres, Ann. Phys. (N.Y.) 64, 599 (1971).

[31] P. C. Peters, Phys. Rev. D 9, 2207 (1974).

[32] M. Giovannini, Classical Quantum Gravity 33, 125002 (2016).

[33] J. Aasi et al. (LIGO Scientific and Virgo Collaborations), Phys. Rev. Lett. 113, 231101 (2014).

[34] B. P. Abbott et al. (LIGO Scientific and Virgo Collaborations), Phys. Rev. Lett. 118, 121101 (2017); 119, 029901(E) (2017).

[35] J. Aasi et al. (LIGO Scientific Collaboration), Classical Quantum Gravity 32, 115012 (2015).

[36] F. Acernese et al. (VIRGO Collaboration), Classical Quantum Gravity 32, 024001 (2015).

[37] Y. Aso, Y. Michimura, K. Somiya, M. Ando, O. Miyakawa, T. Sekiguchi, D. Tatsumi, and H. Yamamoto (KAGRA Collaboration), Phys. Rev. D 88, 043007 (2013).

[38] K. Somiya (KAGRA Collaboration), Classical Quantum Gravity 29, 124007 (2012).

[39] M. Ando et al., Phys. Rev. Lett. 86, 3950 (2001).

[40] B. Willke et al., Classical Quantum Gravity 19, 1377 (2002).

[41] H. Grote (LIGO Scientific Collaboration), Classical Quantum Gravity 27, 084003 (2010).

[42] B. Sathyaprakash et al., Classical Quantum Gravity 29, 124013 (2012); 30, 079501(E) (2013).

[43] P. Amaro-Seoane et al., GW Notes 6, 4 (2013).

[44] G. M. Harry, P. Fritschel, D. A. Shaddock, W. Folkner, and E. S. Phinney, Classical Quantum Gravity 23, 4887 (2006).

[45] S. Kawamura et al., J. Phys. Conf. Ser. 120, 032004 (2008).

[46] S. Kawamura et al., Classical Quantum Gravity 28, 094011 (2011).

[47] M. Abramowitz and I. A. Stegun, Handbook of Mathematical Functions (Dover, New York, 1972).

[48] A. Erdelyi, W. Magnus, F. Obehettinger, and F. Tricomi, Higher Transcendental Functions (McGraw-Hill, New York, 1953).

[49] Y. Zhang, W. Zhao, T. Xia, and Y. Yuan, Phys. Rev. D 74, 083006 (2006).

[50] M. Giovannini, Classical Quantum Gravity 26, 045004 (2009); Phys. Rev. D 82, 083523 (2010).

[51] Y. Cai, Y. T. Wang, and Y. S. Piao, Phys. Rev. D 93, 063005 (2016); 94, 043002 (2016).

[52] V. M. Kaspi, J. H. Taylor, and M. F. Ryba, Astrophys. J. 428, 713 (1994). 
[53] F. A. Jenet, G. B. Hobbs, W. van Straten, R. N. Manchester, M. Bailes, J. P. W. Verbiest, R. T. Edwards, A. W. Hotan, J. M. Sarkissian, and S. M. Ord, Astrophys. J. 653, 1571 (2006).

[54] W. Zhao, Phys. Rev. D 83, 104021 (2011).

[55] P. B. Demorest et al., Astrophys. J. 762, 94 (2013).

[56] W. Zhao, Y. Zhang, X. P. You, and Z. H. Zhu, Phys. Rev. D 87, 124012 (2013).

[57] R. M. Shannon et al., Science 349, 1522 (2015).

[58] V. F. Schwartzmann, JETP Lett. 9, 184 (1969).

[59] M. Giovannini, H. Kurki-Suonio, and E. Sihvola, Phys. Rev. D 66, 043504 (2002).

[60] R. H. Cyburt, B. D. Fields, K. A. Olive, and E. Skillman, Astropart. Phys. 23, 313 (2005).

[61] M. Dentler, A. Hernndez-Cabezudo, J. Kopp, P. Machado, M. Maltoni, I. Martinez-Soler, and T. Schwetz, J. High Energy Phys. 08 (2018) 010.
[62] T. L. Smith, E. Pierpaoli, and M. Kamionkowski, Phys. Rev. Lett. 97, 021301 (2006); I. Sendra and T. L. Smith, Phys. Rev. D 85, 123002 (2012).

[63] M. Giovannini, Classical Quantum Gravity 31, 225002 (2014).

[64] M. Giovannini, Phys. Lett. B 759, 528 (2016).

[65] M. S. Turner, M. J. White, and J. E. Lidsey, Phys. Rev. D 48, 4613 (1993).

[66] S. Chongchitnan and G. Efstathiou, Phys. Rev. D 73, 083511 (2006); Prog. Theor. Phys. Suppl. 163, 204 (2006).

[67] M. Giovannini, Phys. Rev. D 89, 123517 (2014).

[68] Y. Zhang, X. Z. Er, T. Y. Xia, W. Zhao, and H. X. Miao, Classical Quantum Gravity 23, 3783 (2006).

[69] M. Giovannini, Phys. Rev. D 95, 083506 (2017); 96, 101302 (2017).

[70] M. Giovannini, arXiv:1805.08142. 